\title{
Simulation of multi-component multi-phase fluid flow in two-dimensional anisotropic heterogeneous porous media using high-order control volume distributed methods
}

\author{
M. Moshiri ${ }^{\mathrm{a}}$, M.T. Manzari ${ }^{\mathrm{a}, \mathrm{b}, *}$ \\ ${ }^{a}$ School of Mechanical Engineering, Sharif University of Technology, Tehran, Iran \\ ${ }^{b}$ School of Geosciences, University of Aberdeen, Scotland, UK
}

\begin{abstract}
In this paper, flow of multi-component two-phase fluids in highly heterogeneous anisotropic twodimensional porous media is studied using computational methods suitable for unstructured triangular and/or quadrilateral grids. The physical model accounts for miscibility and compressibility of fluids while gravity and capillary effects are neglected. The governing equations consist of a pressure equation together with a system of mass conservation equations. For solving pressure equation, a new method called Control Volume Distributed Finite Element Method (CVDFEM) is introduced which uses Control Volume Distributed (CVD) vertex-centered grids. It is shown that the proposed method is able to approximate the pressure field in highly anisotropic and heterogeneous porous media fairly accurately. The system of mass conservation equations is solved using various upwind and central schemes. These schemes are extended from one-dimensional to two-dimensional unstructured grids. Using a series of numerical test cases, comparison are made between different approaches for approximation of the hyperbolic flux function. Semi one-dimensional high-order data reconstruction procedures are employed to decrease stream-wise numerical diffusion. The results suggest that the Modified Dominant Wave (MDW) scheme outperforms other hyperbolic schemes studied in this paper from both accuracy and computational cost points of view.
\end{abstract}

Keywords: conservation laws, high-order, unstructured, anisotropic, heterogeneous, compositional

\section{Introduction}

Hydrocarbon reservoirs are highly heterogeneous with respect to rock properties, especially permeability for which several orders of magnitude variation within a small distance is not unusual [1]. Such discontinuities in the permeability field will result in strongly discontinuous coefficients when the governing equations are discretized for numerical simulation [2, 3, 4]. However, physical constraints dictate that the flow variables, e.g., pressure and flux, should remain continuous at interfaces of Control Volumes (CV) with discontinuous permeabilities. To obtain local continuity, the Pressure and Flux $(\mathrm{P} / \mathrm{F})$ continuity constraints may be imposed at the discretization level $[2,5]$. In one-dimensional case, this is achieved using well-known harmonic averaging of permeability [6]. On the other hand, in higher-dimensional cases, using the same approach results in first-order diffusion errors [7].

When anisotropy and full-tensor effects are taken into account in higher-dimensions, the numerical scheme should deal with tensorial discontinuous coefficients. Remarkable progress has been made in solving parabolic (or elliptic) pressure equation in anisotropic heterogeneous porous media by introducing the so-called flux-continuous methods such as: Multi-Point Flux Approximation (MPFA) [2, 8, 9], Control Volume Distributed (CVD) Triangular/Full Pressure Support (T/FPS) [7, 10, 11, 12, 13, 14, 15], and Mixed Finite Element Method (MFEM) [16, 17, 18, 19].

\footnotetext{
${ }^{*}$ Corresponding author

Email addresses: moshiri@mech.sharif.edu (M. Moshiri), mtmanzari@sharif.edu (M.T. Manzari)
} 
These methods employ similar approaches to impose $\mathrm{P} / \mathrm{F}$ continuity constraints leading to better accuracy in anisotropic heterogeneous porous media. However, these methods are relatively complicated from implementation point of view and become sensitive to grid geometry when anisotropy ratio between the largest and smallest permeabilities increases. These issues are investigated in this paper using numerical test cases.

On the other hand, in petroleum industry, reservoir data such as rock permeability and porosity, are calculated discretely on separate points and then combined together using geo-statistical models to construct a complete realization of the reservoir and rock properties. This argument favors the use of CVD numerical methods in which the rock properties are assigned to CVs in the dual grid cells. In these methods, the boundaries of different permeability areas are defined after the construction of CVs [7] leading to higher flexibility in handling discontinuous permeabilities.

Similar to the CVD methods, in Control Volume Finite Element (CVFE) method [20, 21], the flow variables are assigned to the CV centers (grid vertices); however, the classical CVFE method differs from the CVD methods as the rock properties are assigned to cells not CVs [20], as a result this method lacks in capturing high contrast permeability discontinuities and tends to smear the flow variables such as saturation or composition [12]. Several investigations are conducted to relieve this smearing of composition by using a combination of higher order finite element with Discontinuous Galerkin (DG) methods [22, 23]. In this paper, a new method is introduced which utilizes some of CVFE concepts but complies with the CVD framework. The proposed Control Volume Distributed Finite Element Method (CVDFEM) is implemented easily and is simple from both conceptual and practical points of view. Here, CVDFEM is compared to the CVD T/FPS methods for solving pressure equation.

In the Implicit Pressure Explicit Composition (IMPEC) procedure, after solving the pressure equation, the solution of hyperbolic system of mass conservation equations should be evaluated explicitly. The latter part has not been investigated as much as the elliptic part. Most of studies use classical upwind schemes to solve for either species concentration in single phase or saturation in two-phase incompressible immiscible flows [13, 9, 24, 25]. When using a compositional model, the number of equations and the computational cost increase dramatically. In this context, the potentials of using central schemes has not been considered to a sufficient degree [26]. This is mainly due to stability and step size limitations of these schemes. However, central schemes take the advantage of symmetric stencil and are more computationally efficient especially when a large system of equations should be solved, which is the case in compositional flows. In this paper, the utilization of central schemes for solving hyperbolic conservation equations associated with the compositional flows is investigated.

When solving conservation equations on higher dimensions, numerical diffusion is usually generated along and across streamlines. This error can be mitigated using spatially high-order numerical schemes. Moreover, using high-order methods with Total Variation Diminishing (TVD) properties lessens the limitations of central schemes. There are several approaches to achieve high-order approximations in unstructured grids $[27,28,13,14]$. Here, one-dimensional high-order reconstruction [29] is extended to a vertex-centered unstructured grid using an edge-based formulation.

To sum up, this paper compares the accuracy and computational cost of several high-order upwind and central numerical schemes in the context of Finite Volume (FV) approach using unstructured grids. Moreover, several CVD methods are evaluated for solving pressure equation in highly heterogeneous anisotropic porous media. This study is conducted for compressible miscible multi-component multi-phase flows in highly heterogeneous anisotropic porous media, while capillary and gravity effects are neglected.

In the following, first a brief description of the physical and mathematical aspects of compositional model is presented. Then, the mathematical formulation of CVD T/FPS and CVDFE methods for solving the pressure equation are introduced. Next, several hyperbolic schemes utilizing high-order data reconstruction for solving mass conservation equations are described. Finally, the capabilities of numerical schemes are assessed by solving several test cases. 


\section{Compositional Model}

In the compositional model used in this paper, reservoir temperature is considered constant and fluid is assumed to be consisted of $n_{c}$ chemical components. Moreover, the fluid has at most $n_{p}=2$ phases, i.e., liquid $l$ and vapor $v$, while capillary and gravity effects are neglected.

Mole number of components is shown by vector $\mathbf{m}$ with $n_{c}$ entities. Assuming thermodynamic equilibrium, components are distributed in the liquid and vapor phases, so that $\mathbf{m}=\mathbf{m}^{l}+\mathbf{m}^{v}$, where $\mathbf{m}^{\alpha}$ is the vector of component mole numbers in phase $\alpha$. Conservation of mass equation for $i$ th component reads

$$
\frac{\partial\left(\phi m_{i}\right)}{\partial t}=-\nabla \cdot \mathbf{u}_{i}+q_{i}, \quad i=1,2, \cdots, n_{c},
$$

where $\phi$ is porosity and $q_{i}$ is source/sink term of component $i$. Also, $\mathbf{u}_{i}$ is defined as,

$$
\mathbf{u}_{i}=-\sum_{\alpha=1}^{n_{p}} x_{i}^{\alpha} \rho^{\alpha} \lambda^{\alpha} \mathbf{K} \nabla p, \quad i=1,2, \cdots, n_{c},
$$

where $p$ is pressure and $\mathbf{K}$ is the tensor of rock absolute permeability. Moreover, $k_{r}^{\alpha}, \mu^{\alpha}$, and $\lambda^{\alpha}=k_{r}^{\alpha} / \mu^{\alpha}$ refer to relative permeability, viscosity, and mobility of phase $\alpha$, respectively. In addition, $x_{i}^{\alpha}$ is mole fraction of component $i$ in phase $\alpha$ and $\rho^{\alpha}$ is molar density of phase $\alpha$. In addition, $\mathbf{K}$ is the tensor of rock absolute permeability and $g$ is the gravitational acceleration.

The pressure equation is obtained using volume balance concept [30] and can be written as

$$
\phi\left(c_{r}+c_{f}\right) \frac{\partial p}{\partial t}=-\sum_{i=1}^{n_{c}} \nu_{i}^{t o t} \nabla \cdot \mathbf{u}_{i}+\sum_{i=1}^{n_{c}} \nu_{i}^{t o t} q_{i},
$$

where $c_{r}$ and $c_{f}$ are rock and fluid compressibilities, respectively and $\nu_{i}^{\text {tot }}$ is total partial molar volume of $i$ th component which is calculated based on Equation Of State (EOS) and Pressure Volume Temperature (PVT) relations [31]. For further discussion on the formulation the reader is referred to [30]. The present paper utilizes SI units.

In this work, an Implicit Pressure Explicit Composition (IMPEC) approach is used to solve the fluid flow equations. In this approach, using the given temperature, pressure, and composition $\mathbf{m}$ at time $t$, first a stability analysis [32] is performed to specify the number of phases that exist in each CV. If both liquid and vapor phases are present, flash calculations [33, 34] should be performed to determine the distribution of components in each phase, i.e., $\mathbf{m}^{\alpha}$. The procedures for calculating thermodynamic equilibrium and fluid properties, e.g. $\rho^{\alpha}$ and $s^{\alpha}$, can be found in $[32,33,35]$. To calculate phase viscosities $\mu^{\alpha}$, an algorithm due to Lohrenz [36] is used which is briefly described in Appendix C.2. Moreover, relative permeabilities $k_{r}^{\alpha}$ are considered to be a function of phase saturation $s^{\alpha}$ only. Using fluid properties, at each time step, pressure equation (3) is solved implicitly while its coefficients are calculated using compositions at present time step. The procedure for solving pressure equation is illustrated in 4.2. Then, using this pressure field, the system of mass conservation equations (1) is solved explicitly to complete a single time step. Solution of these equations is of main concern in this paper and is discussed in 4.3.

\section{Numerical Method}

\subsection{Discretization of Equations}

The numerical scheme used here utilizes a vertex-centered structure $[1,37]$ in which CVs are constructed around grid vertices by connecting cell centroids to edge midpoint as shown in Figure 1. This vertex-centered scheme allows for using computationally efficient edge-wise data structure and flux approximation [38]. Moreover, it facilitates the use of semi- one-dimensional high-order reconstruction [39] as discussed in section 3.3. 


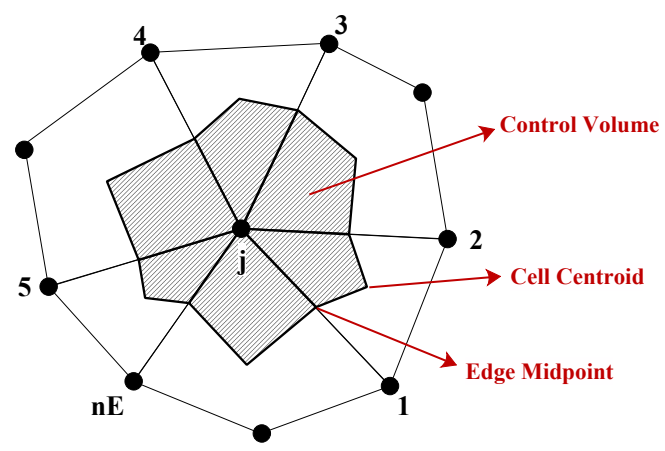

Figure 1: Vertex-centered grid. Grid nodes are shown in black dots. (No. of edges connected to node $j$ is $n_{E}=6$ )

To begin with, the system of conservation equations (1) is integrated over time and $j$ th CV, as shown in Figure 1,

$$
\int_{V_{j}} \int_{t} \frac{\partial\left(\phi m_{i}\right)}{\partial t} \mathrm{~d} t \mathrm{~d} V=-\int_{t} \int_{V_{j}} \nabla \cdot \mathbf{u}_{i} \mathrm{~d} V \mathrm{~d} t+\int_{t} \int_{V_{j}} q_{i} \mathrm{~d} V \mathrm{~d} t, \quad i=1,2, \cdots, n_{c},
$$

using Euler forward time integration together with Gauss divergence theorem for spatial integration and neglecting rock compressibility, the above equation becomes

$$
\frac{V_{j}}{\Delta t} \phi_{j}\left(m_{i, j}^{n+1}-m_{i, j}^{n}\right)=-\oint_{S_{j}} \mathbf{u}_{i}^{n} \cdot \mathbf{n} \mathrm{d} S+Q_{i, j}, \quad i=1,2, \cdots, n_{c},
$$

where $V_{j}$ and $S_{j}$ are volume and surface of $j$ th CV, respectively, and $Q_{i, j} \equiv \int_{V_{j}} q_{i} d V$. Moreover, superscript $n$ refers to time $t_{n}$ and $\Delta t \equiv t_{n+1}-t_{n}$.

The same procedure is applied to the pressure equation (3) except that the time integrations are evaluated implicitly. The final equation takes the form,

$$
\frac{V_{j}}{\Delta t} \phi_{j} c_{f j}\left(p_{j}^{n+1}-p_{j}^{n}\right)=-\oint_{S_{j}} \sum_{i=1}^{n_{c}} \nu_{i}^{t o t} \mathbf{u}_{i}^{n+1} \cdot \mathbf{n} \mathrm{d} S+\sum_{i=1}^{n_{c}} \nu_{i}^{t o t} Q_{i, j} .
$$

Using IMPEC procedure, the numerical method consists of two parts: elliptic part which concerns with solving the pressure equation (6) and hyperbolic part which deals with solving the system of conservation equations (5). The procedure of evaluating integral on the right hand side of (6) in heterogeneous anisotropic media is discussed in section 3.2. The procedures for solving elliptic and hyperbolic parts are discussed in sections 3.2 and 3.3, respectively.

\subsection{Pressure Equation}

To solve the pressure equation, it is necessary to evaluate the flux integral in the pressure equation (6) implicitly. In this paper, the so called, Control Volume Distributed (CVD) approach is used in which the rock properties, e.g., permeability and porosity, are assigned to the center of CVs (grid vertices). Each grid cell consists of $n_{V}$ sub-cells each having a different permeability as illustrated in Figures 2, where $n_{V}$ denotes the number of vertices in each cell (here, 3 or 4 ). Referring to Figure 2, it is seen that

$$
-\oint_{S_{j}} \mathbf{K} \nabla p \cdot \mathbf{n} \mathrm{d} S=-\sum_{e=1}^{n_{E}} \sum_{\alpha=1}^{n_{F E}} \int_{S_{\alpha}} \mathbf{K} \nabla p \cdot \mathbf{n} \mathrm{d} S,
$$

where $n_{E}$ is the number of edges connected to node $j$ and $n_{F E}$ is the number of sub-faces of each edge which is equal to 2 in two-dimensional case. Calculation of right hand side of (7) is then reduced to approximating elliptic fluxes at cross boundaries of CV, i.e.,

$$
F_{\alpha} \equiv-\int_{S_{\alpha}} \mathbf{K} \nabla p \cdot \mathbf{n} \mathrm{d} S
$$


where $S$ and $\mathbf{n}$ are the area and outward normal vector to the sub-cell integration surface. Fluxes of (8) are calculated cell-wise. Hence, $\alpha$ refers to the integral points $(S, E, N, W)$ for quadrilateral and $(S, E, N)$ for triangular cells, respectively. Relocation of these integral points, from cell centroid to edge midpoint, leads to a family of CVD methods as elaborated in [12]. The fluxes of (8) which are approximated within cells, are then assembled to form the fluxes of CV as described in (7). In the following two sections, three approaches are introduced in order to calculate the fluxes implicitly. These methods are especially suitable for simulation of flow in anisotropic fields with heterogeneous permeability.

\subsubsection{Triangular/Full Pressure Support (T/FPS)}

To solve the pressure equation using Triangular/Full Pressure Support (T/FPS) methods [15, 10], Pressure/Flux (P/F) continuity constraints are imposed explicitly at control volume interfaces, i.e., the boundary between gray and white areas of Figure 1 . The problem of imposing $\mathrm{P} / \mathrm{F}$ continuity constraints at CV interfaces is reduced to the imposition of these constraints at the faces of sub-cells within each cell. With reference to Figure 2a, the vector of elliptic fluxes within cell, $\mathbf{F} \equiv\left(F_{S}, F_{E}, F_{N}, F_{W}\right)^{\top}$, can be calculated as $\mathbf{F}_{L} \equiv\left(F_{S}^{1}, F_{E}^{2}, F_{N}^{3}, F_{W}^{4}\right)^{\top}$ or $\mathbf{F}_{R} \equiv\left(F_{S}^{2}, F_{E}^{3}, F_{N}^{4}, F_{W}^{1}\right)^{\top}$ where the subscripts of $F_{I}^{V}$ refer to the integration surfaces $I \equiv(S, E, N, W)$ for quadrilateral and $I \equiv(S, E, N)$ for triangular cells, and superscripts refer to grid vertices $V \equiv\left(1,2, \ldots, n_{V}\right)$ as shown in Figure 2. Both of $\mathbf{F}_{L}$ and $\mathbf{F}_{R}$ can be written as [12]

$$
\begin{aligned}
& \mathbf{F}_{L}=\mathbf{A}_{L} \mathbf{p}_{M}+\mathbf{B}_{L} \mathbf{p}_{V}, \\
& \mathbf{F}_{R}=\mathbf{A}_{R} \mathbf{p}_{M}+\mathbf{B}_{R} \mathbf{p}_{V},
\end{aligned}
$$

where $\mathbf{p}_{M}^{\left(n_{M} \times 1\right)}$ and $\mathbf{p}_{V}^{\left(n_{V} \times 1\right)}$ are pressures at the edge mid-points and centroid of cell $M$ and vertices $V$, respectively. In Figure 2, $M \equiv(s, e, n, w, m)$ for quadrilateral and $M \equiv(s, e, n, m)$ for triangular cells, respectively. In (9a) and (9b), $\mathbf{A}$ and $\mathbf{B}$ refer to coefficient matrices of elliptic fluxes defined in (8). It can be seen that coefficient matrices $\mathbf{A}_{L}$ and $\mathbf{A}_{R}$ are of dimension $\left(n_{M} \times n_{M}\right)$ while $\mathbf{B}_{L}$ and $\mathbf{B}_{R}$ are $\left(n_{M} \times n_{V}\right)$. Continuity of flux at the interface of sub-cells requires that $\mathbf{F}=\mathbf{F}_{L}=\mathbf{F}_{R}$. Mathematically speaking,

$$
\mathbf{F}=\mathbf{A}_{L} \mathbf{p}_{M}+\mathbf{B}_{L} \mathbf{p}_{V}=\mathbf{A}_{R} \mathbf{p}_{M}+\mathbf{B}_{R} \mathbf{p}_{V}
$$

which leads to $n_{V}$ equations and $n_{M}$ unknowns $\mathbf{p}_{M}$. In TPS method, $n_{M}=n_{V}$ so all matrices are of $n_{V} \times n_{V}$ dimension. On the other hand, in FPS method, $n_{M}=n_{V}+1$ and a zero-divergence condition is imposed over an interior auxiliary control-volume surrounding point $m$ to close the system. Mathematically speaking [12]

$$
-\oint_{S_{m}} \mathbf{K} \nabla p \cdot \mathbf{n} \mathrm{d} S=0
$$

Solving for $\mathbf{p}_{M}$ in (10) and defining $\mathbf{C} \equiv \mathbf{A}_{L}\left(\mathbf{A}_{L}-\mathbf{A}_{R}\right)^{-1}\left(\mathbf{B}_{R}-\mathbf{B}_{L}\right)+\mathbf{B}_{L}$, one can obtain [12]

$$
\mathbf{F}=\mathbf{C}^{\left(n_{M} \times n_{V}\right)} \mathbf{p}_{V}^{\left(n_{V} \times 1\right)},
$$

which is the final form of the elliptic flux approximation. Hence, the flux $\mathbf{F}$, depends only on pressures at vertices $\mathbf{p}_{V}$.

\subsubsection{Control Volume Distributed Finite Element Method (CVDFEM)}

Similar to existing CVD T/FPS methods, in CVDFEM, the rock properties are assigned to the center of CVs; however, in order to achieve flux continuity, the permeability tensor is interpolated within each cell in a component-wise manner using standard shape functions of FE. In order for the method to be consistent with the so-called harmonic permeability averaging prevalent in the 


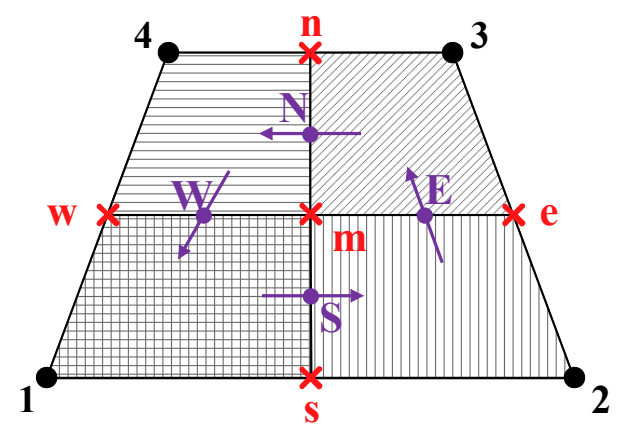

(a) Quadrilateral Cell

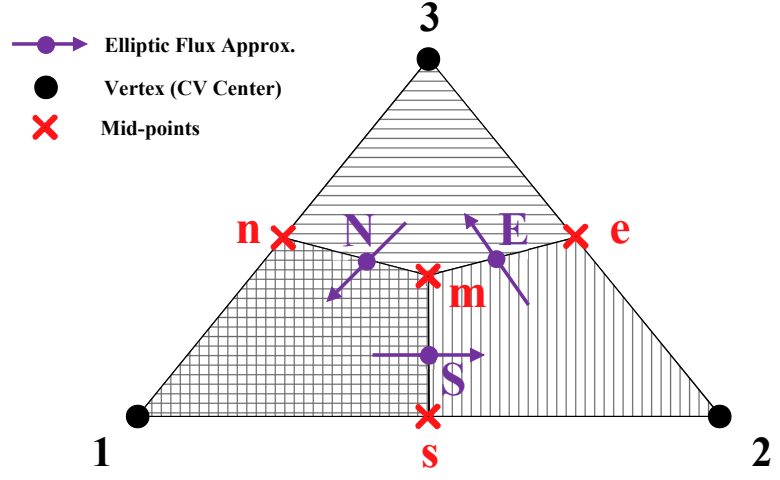

(b) Triangular Cell

Figure 2: Definitions of CVD scheme

literature, the diagonal coefficients of permeability tensor are interpolated inversely while the offdiagonal coefficients are interpolated normally as the latter are zero in most cases. Mathematically speaking,

$$
\frac{1}{K_{i i}}=\sum_{s=1}^{n_{V}} \frac{N_{s}}{K_{i i s}}, \quad \text { and } \quad K_{i j}=\sum_{s=1}^{n_{V}} N_{s} K_{i j_{s}} \quad \text { where } \quad i \neq j .
$$

This leads to a continuous permeability field within each cell, then, the cell fluxes of (8) are approximated within each cell as

$$
\mathbf{F}=\mathbf{D}^{\left(n_{V} \times n_{V}\right)} \mathbf{p}_{V}^{\left(n_{V} \times 1\right)}
$$

In equation (14), $\mathbf{D} \equiv \mathbf{n}^{\top} \mathbf{K} \nabla p$ with $\mathbf{K}$ from (13). Moreover, $\mathbf{F} \equiv\left(F_{S}, F_{E}, F_{N}\right)^{\top}$ and $\mathbf{F} \equiv$ $\left(F_{S}, F_{E}, F_{N}, F_{W}\right)^{\top}$ for triangular and quadrilateral cells, respectively. To be more specific, elliptic flux at integration point $\alpha$ is defined as

$$
F_{\alpha}=\mathbf{n}^{\top} \mathbf{K} \nabla p=\left[\begin{array}{ll}
n_{x} & n_{y}
\end{array}\right]_{\alpha}\left[\begin{array}{ll}
K_{x x} & K_{x y} \\
K_{y x} & K_{y y}
\end{array}\right]_{\alpha}\left[\begin{array}{l}
\frac{\partial p}{\partial x} \\
\frac{\partial p}{\partial y}
\end{array}\right]_{\alpha},
$$

where the $\mathbf{K}$ tensor defined in (13). For the sake of simplicity, Piola-Kirchhoff [12] transformation is used to calculate flux in reference coordination $\xi-\eta$. This transformation for permeability tensor is defined as follow

$$
\tilde{\mathbf{K}}=|J| \mathbf{J}^{-1} \mathbf{K} \mathbf{J}^{-\top},
$$

where $\mathbf{J} \equiv \frac{\partial(x, y)}{\partial(\xi, \eta)}$ is the so-called Jacobian matrix of cell and $J$ is its determinant. Using PiolaKirchhoff transformation results in

$$
F_{\alpha}=\tilde{\mathbf{n}}^{\top} \tilde{\mathbf{K}} \tilde{\nabla} p=\left[\begin{array}{ll}
n_{\xi} & n_{\eta}
\end{array}\right]_{\alpha}\left[\begin{array}{ll}
K_{\xi \xi} & K_{\xi \eta} \\
K_{\eta \xi} & K_{\eta \eta}
\end{array}\right]_{\alpha}\left[\begin{array}{l}
\frac{\partial p}{\partial \xi} \\
\frac{\partial p}{\partial \eta}
\end{array}\right]_{\alpha},
$$

where $\tilde{\mathbf{K}}, \tilde{\nabla} \equiv\left(\frac{\partial}{\partial \xi}, \frac{\partial}{\partial \eta}\right)^{\top}$, and $\tilde{\mathbf{n}} \equiv\left(n_{\xi}, n_{\eta}\right)^{\top}$ are counterparts of $\mathbf{K}, \nabla \equiv\left(\frac{\partial}{\partial x}, \frac{\partial}{\partial y}\right)^{\top}$, and $\mathbf{n} \equiv\left(n_{x}, n_{y}\right)^{\top}$ in local coordinates $\xi-\eta$ defined in Figure 3. Moreover, the position in the physical domain is related to the reference coordinates as

$$
x=\sum_{j=1}^{n_{V}} N_{j}(\xi, \eta) x_{j}, \quad y=\sum_{j=1}^{n_{V}} N_{j}(\xi, \eta) y_{j} .
$$

In relation (17), derivateves of pressure are calculated as

$$
\frac{\partial p}{\partial \xi}=\sum_{j=1}^{n_{V}} \frac{\partial N_{j}(\xi, \eta)}{\partial \xi} p_{j}, \quad \frac{\partial p}{\partial \eta}=\sum_{j=1}^{n_{V}} \frac{\partial N_{j}(\xi, \eta)}{\partial \eta} p_{j} .
$$




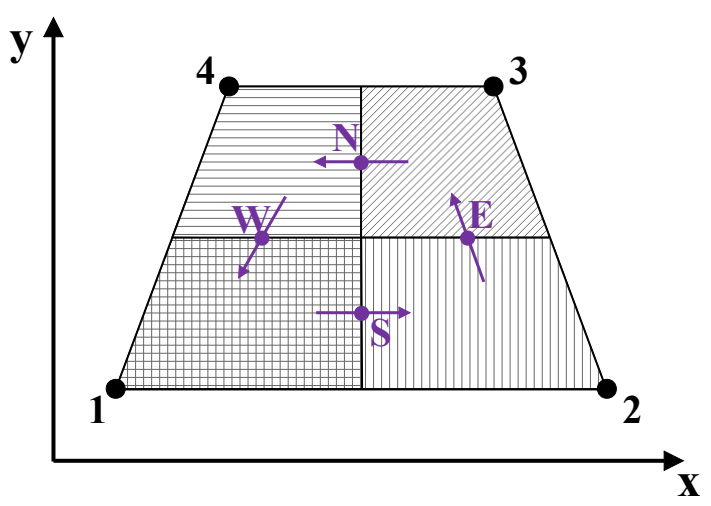

(a) Cell in physical domain

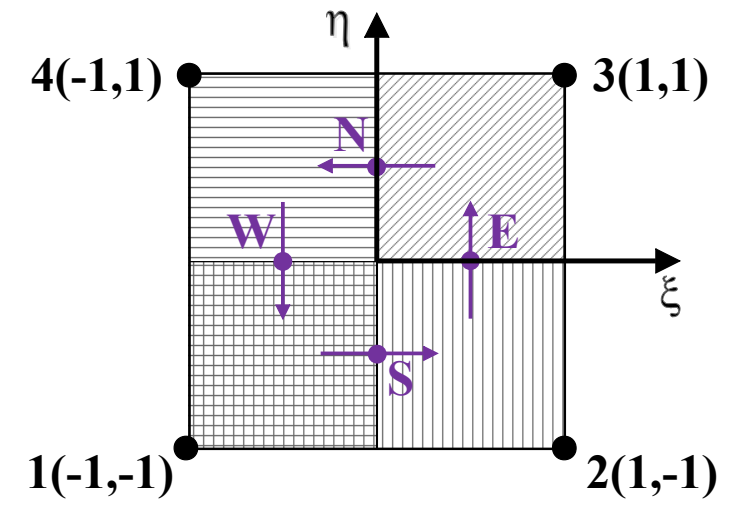

(b) Cell in computational domain

Figure 3: Transformation between physical and computational domains for CVDFE method

and $(\tilde{\mathbf{n}})_{\alpha}$ of reference cell can be easily defined as follow

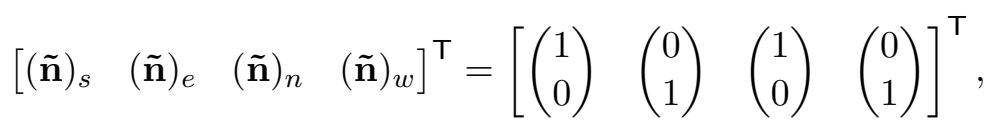

for quadrilateral cells, and similar relations can be obtain for triangular cells.

To sum up, in CVDFEM the continuity of rock properties is assumed which automatically leads to pressure and flux continuities. The reason for the above averaging choice is discussed below. First, it is necessary for the new CVDFE method to be consistent with conventional harmonic averaging of permeability when diagonal permeability is used in Cartesian grid, so the diagonal permeability coefficients shall be interpolated such that they can be reduced to conventional harmonic averaging. Second, the proposed interpolation shall be able to work with zero off-diagonal coefficients which is the case in many situations and the proposed interpolation procedure avoids division by zero problem.

It is worth mentioning that, TPS, FPS, and other explicit flux continuous methods, in fact, alter the coefficient tensor $\mathbf{C}$ in flux calculation to achieve pressure continuity using different manipulations, while the newly proposed CVDFE method modifies the $\mathbf{K}$ tensor itself within each cell to provide flux continuity. In section 4 , the results of CVDFE method are compared with those of CVD T/FPS methods in heterogeneous anisotropic test cases. Moreover, authors' experiences show that CVDFEM can perform better in difficult situations where the full-tensor anisotropy or heterogeneity are high. It should be noted that the conservativeness of CVDFE method has not been proved rigorously yet, but in the problems studied by the authors this did not affect the stability or accuracy of the solution.

As it can be seen form equation (7), $F_{\alpha}$ as defined in (8) is just the elliptic flux while the total flux $f=\mathbf{f}^{H} F$ including hyperbolic fluxes $\mathbf{f}^{H}$ of components as well. The procedure for calculation of latter will be clarified in the next section.

\subsection{Conservation Equations}

To solve the system of conservation equations (5) an edge-wise approach is used. Firstly, as shown in Figure 4a, the elliptic flux of each edge $\left(F_{e}\right)$ is calculated by adding up elliptic sub-face fluxes (as shown in Figures $2 \mathrm{a}$ and $2 \mathrm{~b}$ ) from equations (12) or (14), i.e., $F_{e}=\sum_{\alpha=1}^{n_{F E}} F_{\alpha}$. Secondly, hyperbolic flux $\mathbf{f}^{H}=\sum_{\alpha=1}^{n_{p}} x_{i}^{\alpha} \rho^{\alpha} \lambda^{\alpha}$ is calculated at left $L$ and right $R$ states of each edge, as depicted in Figure 4b. Thirdly, the total flux is calculated using $\mathbf{f}_{L}=\mathbf{f}_{L}^{H} F_{e}$ and $\mathbf{f}_{R}=\mathbf{f}_{R}^{H} F_{e}$ at the left and right states, respectively; in which $\mathbf{f}_{L}^{H}=\mathbf{f}^{H}\left(\mathbf{m}_{L}\right)$ and $\mathbf{f}_{R}^{H}=\mathbf{f}^{H}\left(\mathbf{m}_{R}\right)$ are hyperbolic flux 
functions calculated at the left and right states of edge $e$, respectively. Finally, (5) is rewritten as

$$
\frac{V_{j}}{\Delta t} \phi_{j}\left(\mathbf{m}_{j}^{n+1}-\mathbf{m}_{j}^{n}\right)=\sum_{e=1}^{n_{E}} \hat{\mathbf{f}}_{e}^{n}+\mathbf{Q}_{j},
$$

where $\mathbf{Q}_{j}$ is the source/sink term and $\hat{\mathbf{f}}_{e}$ is the so-called numerical flux which is calculated using $\mathbf{f}_{L}$ and $\mathbf{f}_{R}$ by any of the procedures described in this section. In (21), the edge numerical flux $\hat{\mathbf{f}}_{e}$ is assumed positive when it enters $j$ th $\mathrm{CV}$ as shown in Figure 4c. Hereafter, the subscript $e$ is omitted for brevity. There are a number of options to approximate $\hat{\mathbf{f}}_{e}$ among which four schemes are presented here: ROE, HLL, LLF, and MDW. For further discussion on these schemes consult $[29,39]$.

\subsubsection{Roe's Upwind Scheme (ROE)}

ROE scheme is a modification of the well-known Roe's upwind scheme [40] in which all wave directions are computed via a characteristic decomposition. Mathematically speaking, numerical flux of (21) is defined as,

$$
\hat{\mathbf{f}}=\frac{1}{2}\left(\mathbf{f}_{R}+\mathbf{f}_{L}\right)-\frac{1}{2} \mathbf{R}|\boldsymbol{\Lambda}| \mathbf{R}^{-1}\left(\mathbf{m}_{R}-\mathbf{m}_{L}\right)
$$

where $\boldsymbol{\Lambda} \equiv \operatorname{diag}\left(\lambda_{1}, \lambda_{2}, \cdots, \lambda_{n_{c}}\right)$ while $\lambda_{j}$ is the $j$ th eigenvalue and $\mathbf{R}=\left[\mathbf{r}_{1}, \mathbf{r}_{2}, \cdots, \mathbf{r}_{n_{c}}\right]$ is the matrix of right eigenvectors of the Jacobian matrix $\frac{1}{\phi} \frac{\partial \mathbf{f}}{\partial \mathbf{m}}$ evaluated at $\mathbf{m}_{M}=\frac{\mathbf{m}_{L}+\mathbf{m}_{R}}{2}$. It should be noted that, in order to use this scheme, it is necessary to have a set of independent eigenvectors so that $\mathbf{R}^{-1}$ can be calculated, otherwise at umbilic points (where two or more eigenvalues are equal and loss in eigenvector basis occurs) the scheme may fail to produce entropy satisfying solution. As the procedures for dealing with problematic points are relatively complex in the original Roe's scheme, a simplified procedure is introduced in Section 3.5.

\subsubsection{Harten-Lax-van Leer Scheme (HLL)}

The HLL scheme is a two-wave scheme that theoretically lies between the upwind and central schemes. Numerical flux is defined as [41],

$$
\hat{\mathbf{f}}= \begin{cases}\mathbf{f}_{L} & \tilde{\lambda}_{L} \geq 0 \\ \frac{\tilde{\lambda}_{R} \mathbf{f}_{L}-\tilde{\lambda}_{L} \mathbf{f}_{R}+\tilde{\lambda}_{L} \tilde{\lambda}_{R}\left(\mathbf{m}_{R}-\mathbf{m}_{L}\right)}{\tilde{\lambda}_{R}-\tilde{\lambda}_{L}} & \tilde{\lambda}_{L}<0<\tilde{\lambda}_{R}, \\ \mathbf{f}_{R} & \tilde{\lambda}_{R} \leq 0\end{cases}
$$

where $\tilde{\lambda}_{L}$ and $\tilde{\lambda}_{R}$ are given in Appendix A. As it can be seen from equation (23), this scheme requires several eigen-structure calculations to approximate wave speeds, and as a result its computational cost is relatively high.

\subsubsection{Local Lax-Friedrichs Scheme (LLF)}

Central schemes are a viable alternative for solving hyperbolic equations which do not require characteristic decomposition. LLF scheme lies in this category in which the term $\mathbf{R}|\boldsymbol{\Lambda}| \mathbf{R}^{-1}$ is approximated by $\left|\lambda_{L L F}\right|$ I. Mathematically speaking [42],

$$
\hat{\mathbf{f}}=\frac{1}{2}\left(\mathbf{f}_{R}+\mathbf{f}_{L}\right)-\frac{1}{2}\left|\lambda_{L L F}\right| \mathbf{I}\left(\mathbf{m}_{R}-\mathbf{m}_{L}\right),
$$

where $\left|\lambda_{L L F}\right|=\max \left(\left|\lambda_{L}^{\max }\right|,\left|\lambda_{M}^{\max }\right|,\left|\lambda_{R}^{\max }\right|\right)[42]$. Moreover, $\lambda_{L}^{\max }, \lambda_{M}^{\max }$, and $\lambda_{R}^{\max }$ are the maximum of absolute eigenvalues at Left, Right, and Mid-points, which are calculated at $\mathbf{m}_{L}, \mathbf{m}_{R}$, and $\mathbf{m}_{M}$, respectively. $\mathbf{I}$ is the identity matrix. 


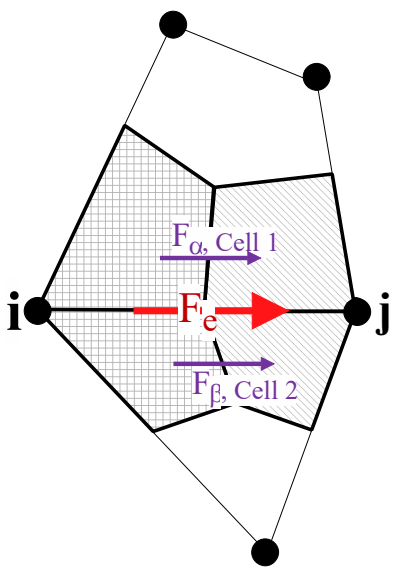

(a) Elliptic flux

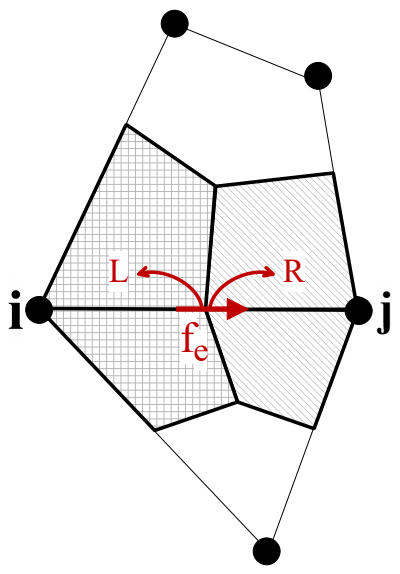

(b) Hyperbolic flux

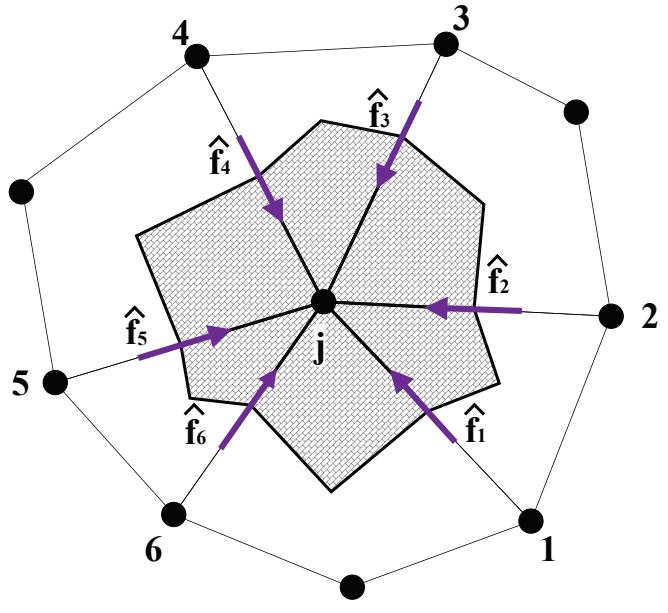

(c) Positive sign convention

Figure 4: Definition of fluxes in CVD methods

\subsubsection{Modified Dominant Wave Scheme (MDW)}

While the LLF scheme is decomposition free, it is still complicated and several eigen-structures should be evaluated at each interface. Modified Dominant Wave (MDW) scheme alleviates the aforementioned problem by using a simple formula to evaluate the wave speed without using an eigen-structure calculation. i.e.,

$$
\hat{\mathbf{f}}=\frac{1}{2}\left(\mathbf{f}_{R}+\mathbf{f}_{L}\right)-\frac{1}{2}\left|\lambda_{M D W}\right| \mathbf{I}\left(\mathbf{m}_{R}-\mathbf{m}_{L}\right),
$$

where dominant wave speed is defined as [39],

$$
\left|\lambda_{M D W}\right|=\max \left(\left|\bar{\lambda}_{L-G L}\right|,\left|\bar{\lambda}_{G L-M}\right|,\left|\bar{\lambda}_{M-G R}\right|,\left|\bar{\lambda}_{G R-R}\right|\right),
$$

and

$$
\bar{\lambda}_{1-2}=\frac{\left(\mathbf{m}_{2}-\mathbf{m}_{1}\right) \cdot\left(\mathbf{f}_{2}-\mathbf{f}_{1}\right)}{\left(\mathbf{m}_{2}-\mathbf{m}_{1}\right) \cdot\left(\mathbf{m}_{2}-\mathbf{m}_{1}\right)} .
$$

This definition helps particularly when flux functions are linearly degenerate [42]. The latter scheme has the advantage of not being prone to entropy violating solutions while the added computational cost is relatively small.

It should be noted that, $L$ and $R$ states are the same as $i$ and $j$ in the first-order schemes, but when high-order schemes are used these states depend on a larger stencil, as illustrated in the next section.

\subsection{High-order Methods}

Using high-order accuracy in space is known to improve the resolution, especially in onedimensional problems. Here, the edge-based data structure allows to reconstruct high-order variables in a semi-one-dimensional manner. The only difference is that the search for extended points is not a trivial task because of geometrical complexities in unstructured grids. Several approaches were investigated to construct the computational stencil [43], i.e., $I$ and $J$ associated with edge $e$. Here, the corresponding edge is extended from both ends to reach the opposite sides of the nearest triangles as shown in Figure 5. The position of these extended points should be found for all edges. The fluid states at these extended points should be obtained using an appropriate approximation. For example, the field value $\psi$ at any point within a triangle can be calculated using a simple Finite Element interpolation as

$$
\psi=\sum_{k=1}^{3} N_{k}(\xi, \eta) \psi_{k},
$$




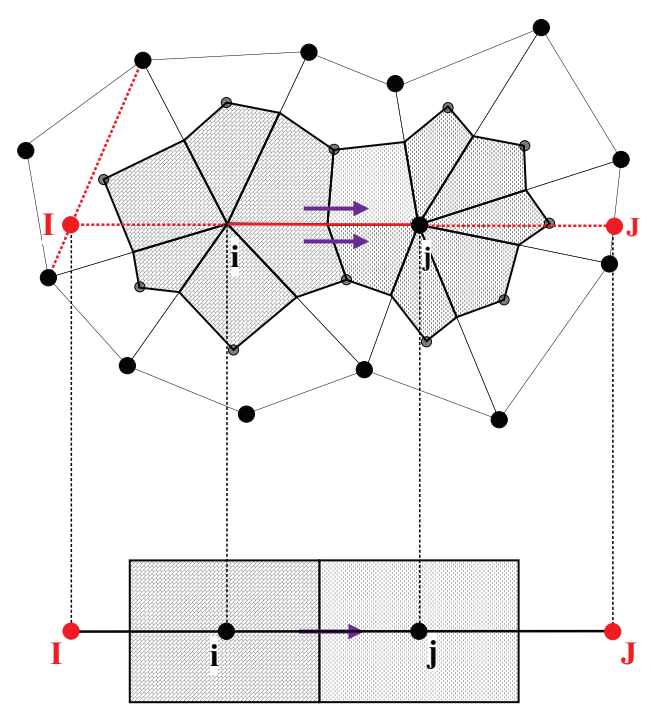

Figure 5: high-order points in unstructured grid

where $N_{k}$ is the $k$ th shape function and $\psi_{k}$ is the corresponding field data at nodal points while $\xi$ and $\eta$ are the local coordinates of the triangle. First-order states are substituted whenever either of points $i$ or $j$ are located on the boundaries ( $I$ or $J$ are located outside the domain). Finally the required states in the semi-1D stencil $(I, i, j, J$ in Figure 5$)$ are used to reconstruct high-order states similar to one-dimension [29,39]. To recover the first-order schemes, $L$ and $R$ states of each edge are simply the values of neighboring nodes, i.e., $i$ and $j$.

In this paper, two procedures are used to reconstruct high-order variables from the extended points: 1) Monotone Upstream-centered Schemes for Conservation Laws (MUSCL) and 2) Weighted Essentially Non Oscillatory (WENO) reconstructions which are briefly described in the following sections. It should be noted that, the procedures described here are limited to second-order spatial accuracy.

\subsubsection{MUSCL Reconstruction}

In order to use a MUSCL high-order reconstruction [44], $L$ and $R$ states are defined as [45],

$$
\begin{aligned}
& m_{L}=m_{i}+\frac{1}{2} \varphi\left(r^{+}\right)\left(m_{j}-m_{i}\right), \\
& m_{R}=m_{j}-\frac{1}{2} \varphi\left(r^{-}\right)\left(m_{j}-m_{i}\right),
\end{aligned}
$$

respectively, all computed for edge $e$. In equations (29), $m$ denotes a typical component of vector $\mathbf{m}$ while $\varphi\left(r^{+}\right)$and $\varphi\left(r^{-}\right)$are the slope limiters in which

$$
\begin{aligned}
& r^{+}=\frac{m_{i}-m_{I}}{m_{j}-m_{i}} \cdot \frac{\left|\mathbf{r}_{j}-\mathbf{r}_{i}\right|}{\left|\mathbf{r}_{i}-\mathbf{r}_{I}\right|}, \\
& r^{-}=\frac{m_{J}-m_{j}}{m_{j}-m_{i}} \cdot \frac{\left|\mathbf{r}_{j}-\mathbf{r}_{i}\right|}{\left|\mathbf{r}_{J}-\mathbf{r}_{j}\right|},
\end{aligned}
$$

where $\mathbf{r}_{k}$ is the position vector for $k=I, i, j, J$ points, as defined in Figure 5 . In this paper, minmod limiter $\varphi(r)=\min (r, 1)$ is used. For further alternatives consult [46, 47].

\subsubsection{WENO Reconstruction}

For the second-order WENO reconstruction, $m_{L}$ and $m_{R}$ are defined as [48]

$$
\begin{aligned}
& m_{L}=w_{0}\left(\frac{1}{2} m_{i}+\frac{1}{2} m_{j}\right)+w_{1}\left(-\frac{1}{2} m_{I}+\frac{3}{2} m_{i}\right), \\
& m_{R}=w_{2}\left(\frac{1}{2} m_{i}+\frac{1}{2} m_{j}\right)+w_{3}\left(-\frac{1}{2} m_{J}+\frac{3}{2} m_{j}\right),
\end{aligned}
$$


for edge $e$, where $w_{0}$ to $w_{3}$ are weight functions described in [48]. A brief review of this procedure is given in Appendix $\mathrm{B}$.

\subsection{Correction for Stagnation and Umbilic Points}

To prevent unphysical entropy violating solutions when upwind schemes are used, a modified version of LLF scheme is used in problematic points as follow [42]:

$$
\hat{\mathbf{f}}= \begin{cases}\hat{\mathbf{f}}_{L L F} & \lambda_{k L} \leq 0 \leq \lambda_{k R}, \quad k=1,2, \cdots, n_{c}, \\ \hat{\mathbf{f}}_{L L F} & \min \left(\left|\lambda_{i}-\lambda_{j}\right|\right)<\varepsilon\left(\max _{k}\left|\lambda_{k}\right|\right), \quad i, j, k=1,2, \cdots, n_{c}, \quad i \neq j, \\ \hat{\mathbf{f}}_{\text {original }} & \text { otherwise }\end{cases}
$$

Numerical experiences show that 0.01 is an appropriate value for $\varepsilon$ [29]. The first line in (32) refers to stagnation points, while the second line describes the umbilic points where eigenvector deficiency may occur as a consequence of equal eigenvectors.

\subsection{Time Step Size}

To calculate time step size, positivity and stability concepts are utilized. Specifically, to obtain a non-negative $m_{i}$ in the new time step, with reference to Figure 4c, the mole number of $i$ th component in $j$ th CV (i.e., $\phi_{j} V_{j} m_{i, j}$ ) shall be greater than the mole number leaving the $\mathrm{CV}$ in a single time step (i.e., $-\Delta t \sum_{e=1}^{n_{E}} \hat{f}_{i, e}^{-}$). To achieve a global time step size, the same requirement shall be satisfied for all flux components in each CV. Mathematically speaking [49],

$$
\Delta t=\mathrm{CFL} \min _{j=1}^{n_{C V}}\left[\min _{i=1}^{n_{c}}\left(-\frac{\phi_{j} V_{j} m_{i, j}}{\sum_{e=1}^{n_{E}} \hat{f}_{i, e}^{-}}\right)\right]
$$

where $\hat{f}_{i, e}^{-} \equiv 0.5\left(\hat{f}_{i, e}-\left|\hat{f}_{i, e}\right|\right)$ is the numerical flux of $i$ th component leaving $j$ th CV in $n$th time step and CFL denotes Courant-Friedrichs-Lewy number. Time step size obtained from (33) leads to a stable solution; however, a user-defined threshold on the $m_{i, j}$ should be provided in order to avoid infinitely small time step size. To prevent this problem, in this paper, the following alternative formula is suggested

$$
\Delta t=\mathrm{CFL} \underset{j=1}{\min _{C V}}\left[\min _{i=1}^{n_{c}}\left(-\frac{\phi_{j} V_{j}}{\sum_{e=1}^{n_{E}} \lambda_{i, e}^{-}}\right)\right]
$$

where $\lambda_{i, e}^{-} \equiv 0.5\left(\lambda_{i, e}-\left|\lambda_{i, e}\right|\right)$ while $\lambda_{i, e}$ is the $i$ th eigenvalue of the hyperbolic system calculated for edge $e$.

\section{Results}

In this section, several test cases are solved to demonstrate the accuracy and computational performance of the numerical procedures presented in this paper. All test cases are solved on two-dimensional unstructured grid. The results are presented in three sections: 1) validation of numerical procedures, 2) evaluation of procedures for solving pressure equation, and 3) evaluation of schemes for solving mass conservation equations.

\subsection{Homogeneous Anisotropic Five-spot Problem}

For the sake of validation, in this section, proposed numerical procedures of this paper is compared with compositional commercial reservoir simulator Eclipse E300 (Schlumberger 2014). A quarter of five spot test case is considered for this comparison. In this two-component problem, reservoir is initially filled with $\mathrm{nC}_{10}$ in liquid and $\mathrm{C}_{1}$ in vapor phase is injected from bottom left corner while liquid is produced from top right corner. Constant volumetric flow rate constraint is used for the injection well while the so-called Bottom Hole Pressure (BHP) of the production well is held constant. Required properties are given Table 4. To compare the result of vertexcentered procedure of current study with cell-centered method of E300 software, the outermost 
layer of cells are chosen to be half of interior ones in E300 model. The geometry of domain and positions of injection and production wells are given in Figure 6a. Anisotropic diagonal permeability $\mathbf{K}=\operatorname{diag}(100,10) \mathrm{mD}$ is considered for this test case with $3280 \times 3280 \mathrm{ft}^{2}$ computational domain.

In order to quantitatively compare various methods for solving pressure equation, first, the problem is solved on a $20 \times 20$ uniform Cartesian grid with E300 simulator. Second, the grid is distorted and/or refined and the same problem is solved on highly distorted grids. Reference solutions for production CV pressure and recovery of liquid hydrocarbon (oil) versus time are presented in Figures $6 \mathrm{~b}$ and 6c, respectively. MDW scheme is utilized to solve conservation equation and CFL is set to 0.6 according to equation (34) while using other hyperbolic schemes does not change the results considerably. The pressure part is solved using CVDTPS, CVDFPS, and CVDFE methods on six various computational grids and the results are compared with E300 simulator result using appropriate error norms. The error norm is defined as

$$
E=\frac{\left[\sum_{i=1}^{n_{\text {step }}}\left(\psi_{i} \Delta t_{i}-\psi_{i}^{r e f} \Delta t_{i}\right)^{2}\right]^{1 / 2}}{\left[\sum_{i=1}^{n_{\text {step }}}\left(\psi_{i}^{r e f} \Delta t_{i}\right)^{2}\right]^{1 / 2}} .
$$

where $\psi_{i}$ is the variable and $\psi_{i}^{r e f}$ is the reference value of variable. Moreover, $\Delta t_{i}$ is the $i$ th time step size and $n_{\text {step }}$ denotes the number of time steps. Figure 7 shows the grids used in this problem. Figure 7a depicts $20 \times 20$ uniform Cartesian grid similar to that of E300 model. Figure 7b and 7b are two highly distorted quadrilateral grids with 400 computational nodes. Moreover, Figure 7d, 7e, and $7 \mathrm{f}$ show three unstructured triangular grids. In Figure 8a, the error norms of oil recovery is depicted with respect to different pressure methods and various grids. Moreover, in Figure 8b, the error norms of pressure of production $\mathrm{CV}$ is depicted with respect to various methods. Tables 1 and 2 show the aforementioned error norms associated to oil recovery and pressure of production $\mathrm{CV}$, respectively. It can be seen that, in all cases results of CVDFE and CVDFPS methods are comparable and outperform CVDTPS method from accuracy point of view.

Moreover, it can be shown that, for triangular grids, the new CVDFE produces the same results as the more complicated CVDFPS method whenever diagonal homogeneous permeability field is simulated similar to the one considered in this section.

Table 1: Relative error norm of oil recovery for first test case

\begin{tabular}{|c|c|c|c|}
\hline & TPS & FPS & CVDFE \\
\hline Grid I & 0.7020 & 0.7069 & 0.7069 \\
\hline Grid II & 1.8132 & 1.6087 & 1.5984 \\
\hline Grid III & 2.1449 & 1.8166 & 1.7744 \\
\hline Grid IV & 3.0756 & 1.5045 & 1.5045 \\
\hline Grid V & 3.4086 & 1.1562 & 1.1562 \\
\hline Grid VI & 5.1108 & 4.1291 & 4.1291 \\
\hline
\end{tabular}

Table 2: Relative error norm of production CV pressure for first test case

\begin{tabular}{|c|c|c|c|}
\hline & TPS & FPS & CVDFE \\
\hline Grid I & 0.1254 & 0.1210 & 0.1210 \\
\hline Grid II & 0.3067 & 0.2410 & 0.2406 \\
\hline Grid III & 0.3186 & 0.2471 & 0.2483 \\
\hline Grid IV & 0.5946 & 0.2846 & 0.2846 \\
\hline Grid V & 0.5997 & 0.2889 & 0.2889 \\
\hline Grid VI & 0.6562 & 0.2883 & 0.2883 \\
\hline
\end{tabular}




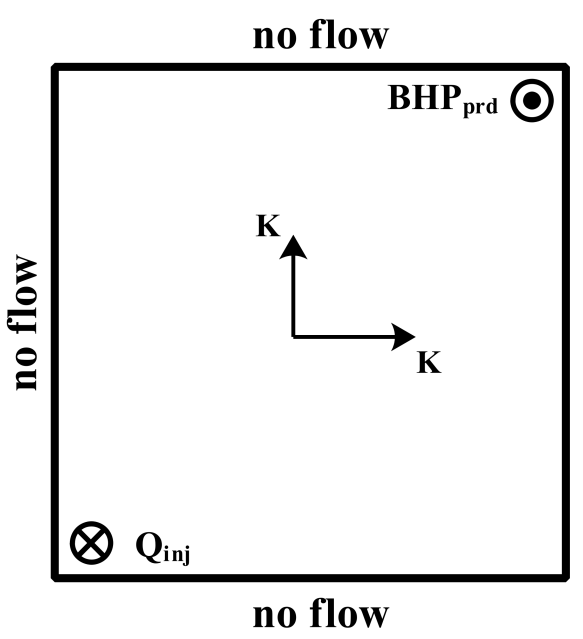

(a) Geometry and well location

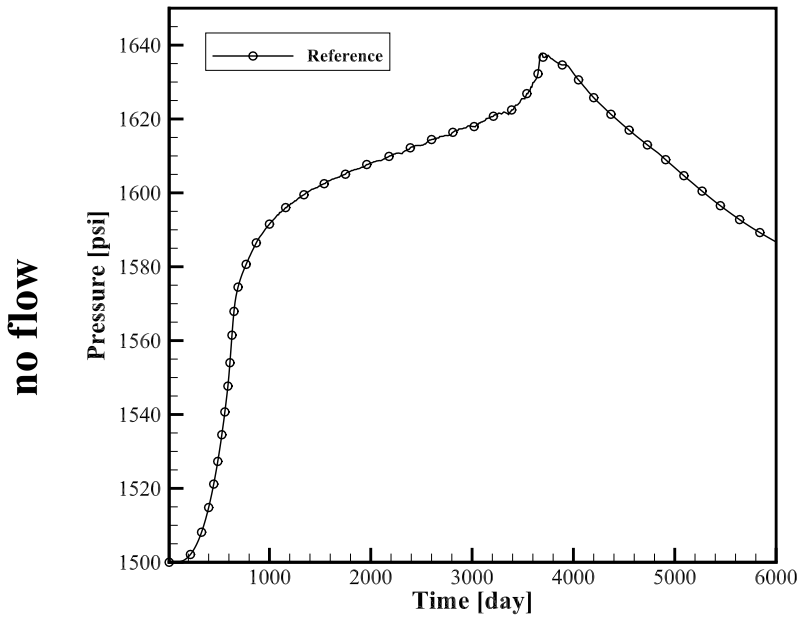

(b) Pressure of production CV

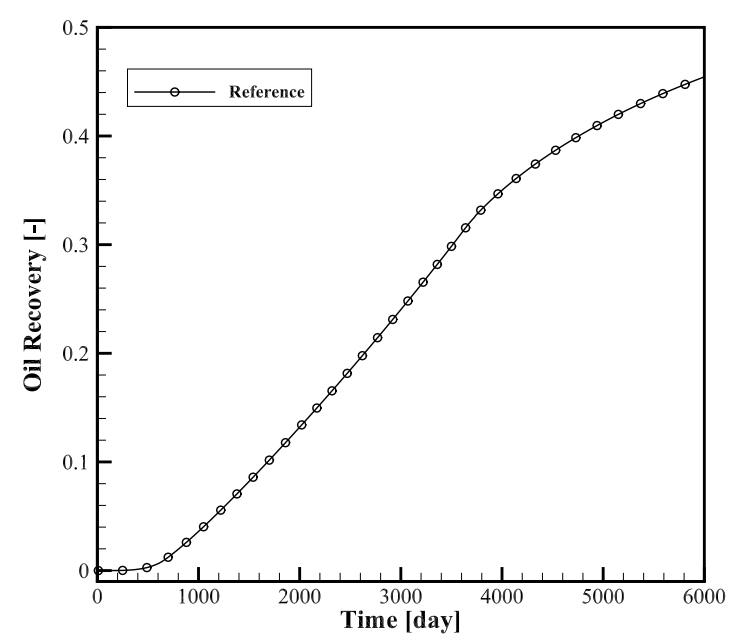

(c) recovery of liquid hydrocarbon

Figure 6: Reservoir geometry and reference solution of anisotropic homogeneous five-spot problem 


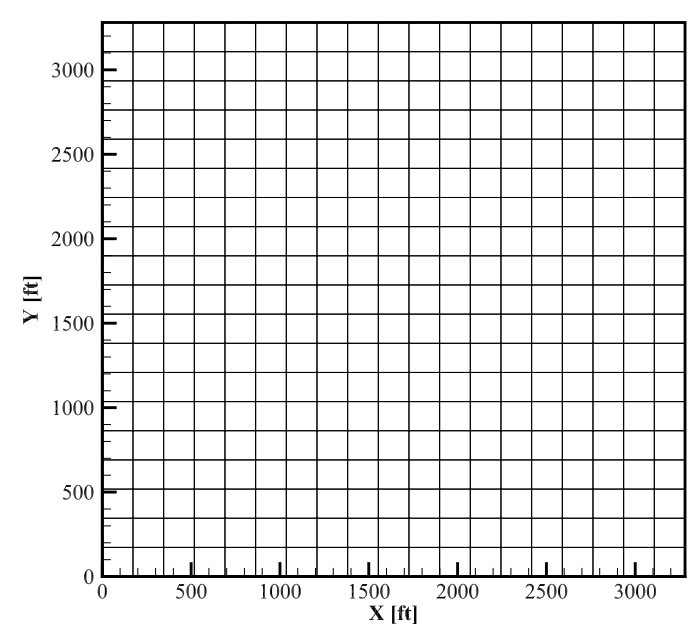

(a) Grid I

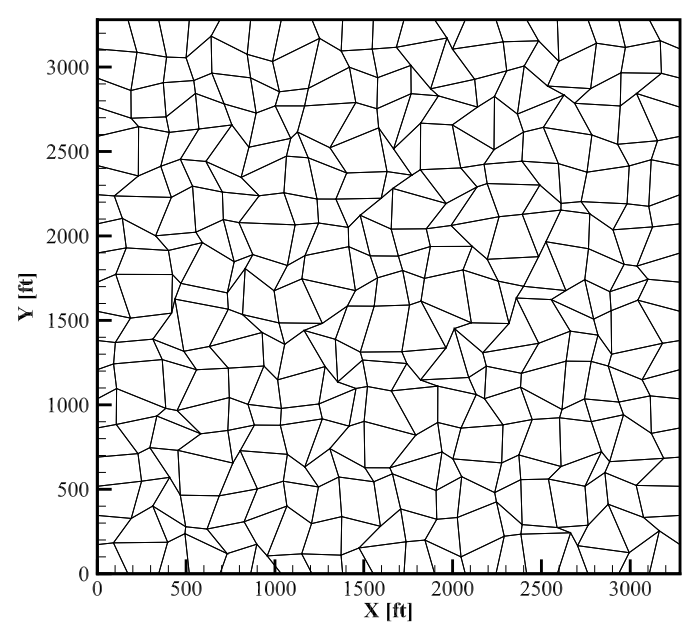

(c) Grid III

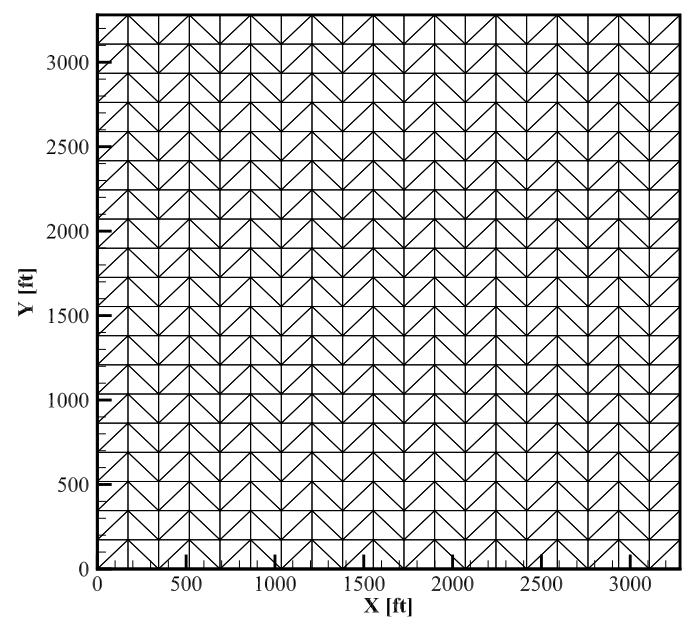

(e) Grid V

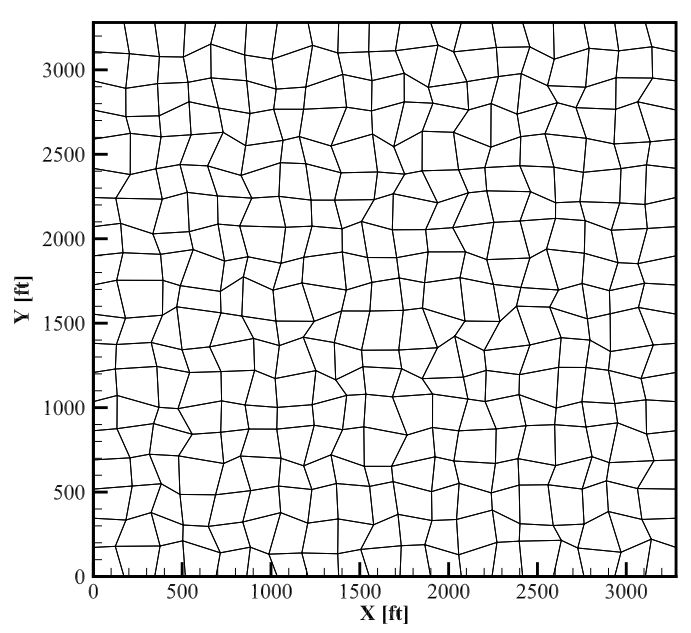

(b) Grid II

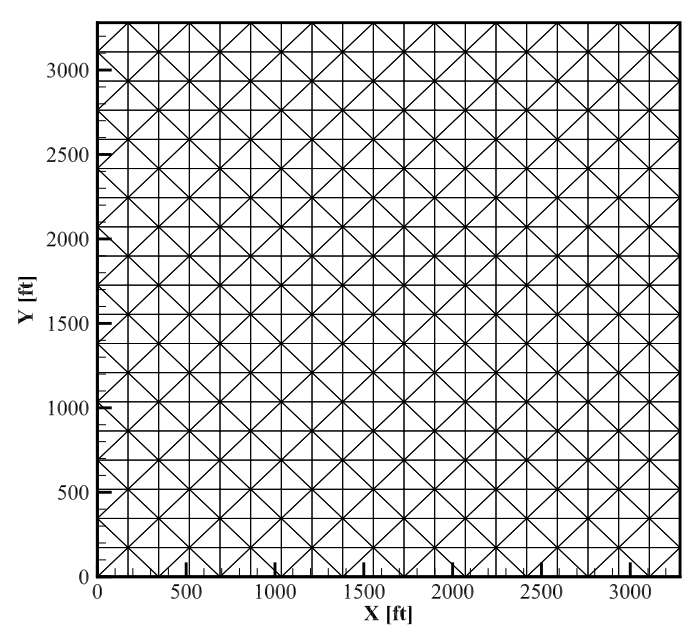

(d) Grid IV

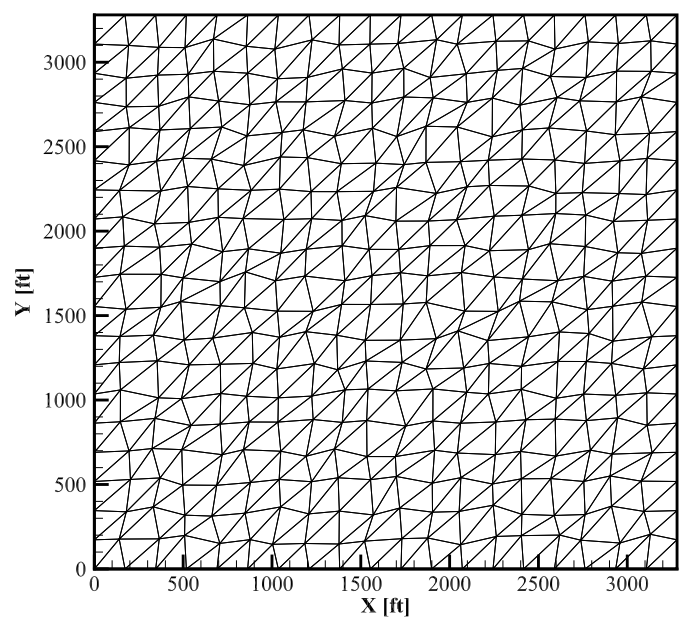

(f) Grid VI

Figure 7: Various grids used in first test case 


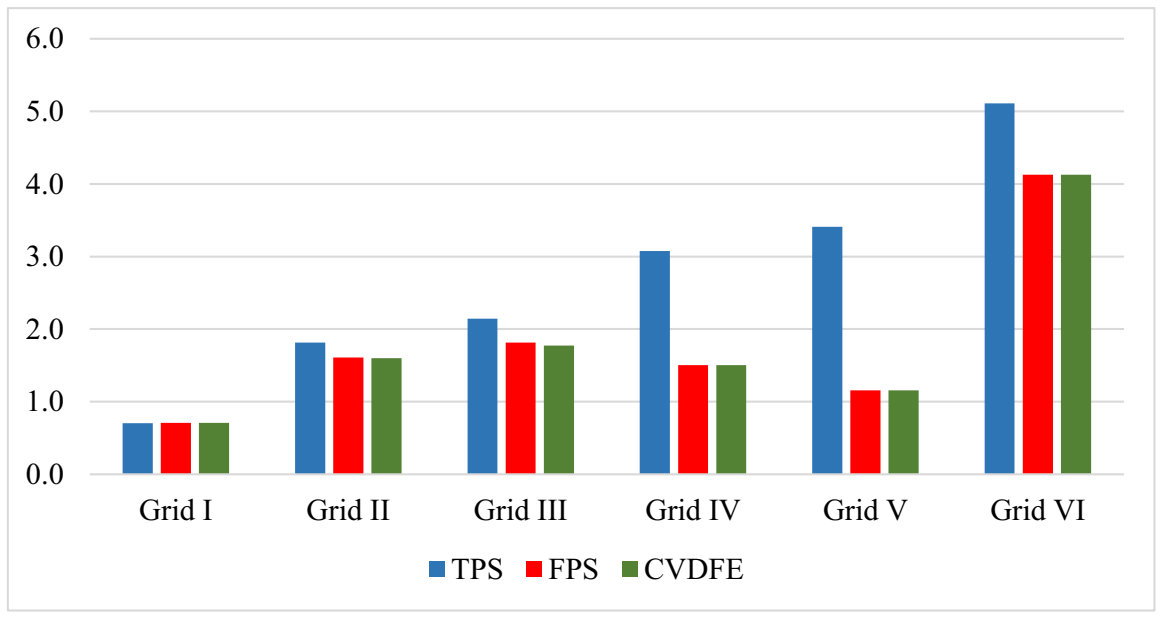

(a) Oil recovery error (-)

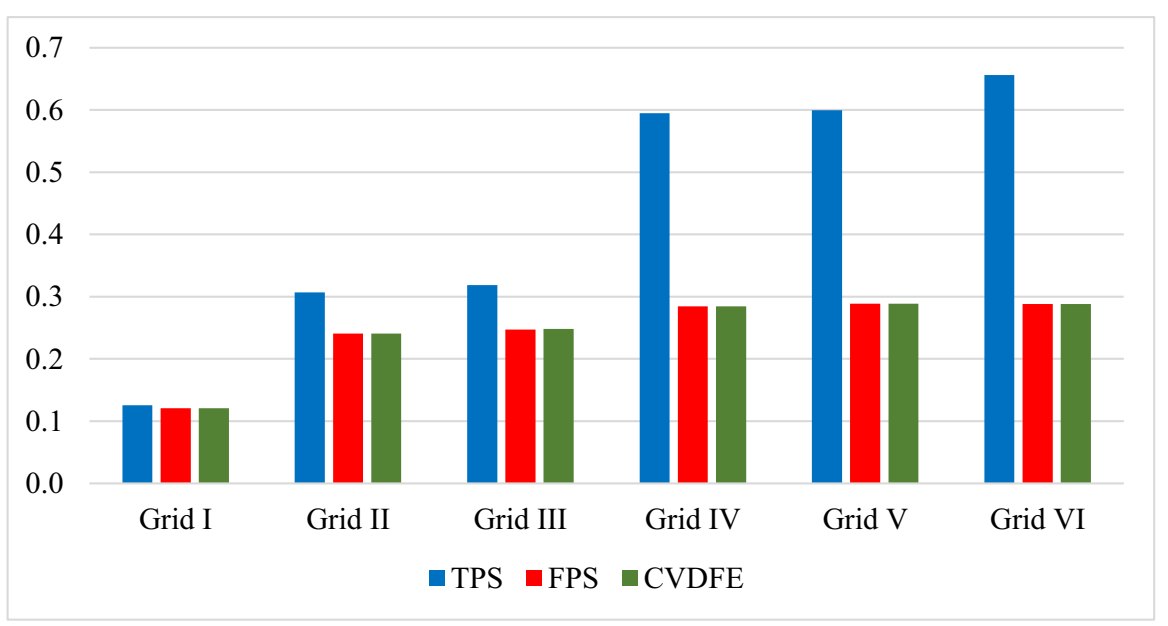

(b) Error in pressure of production CV (-)

Figure 8: Relative error of first test case 


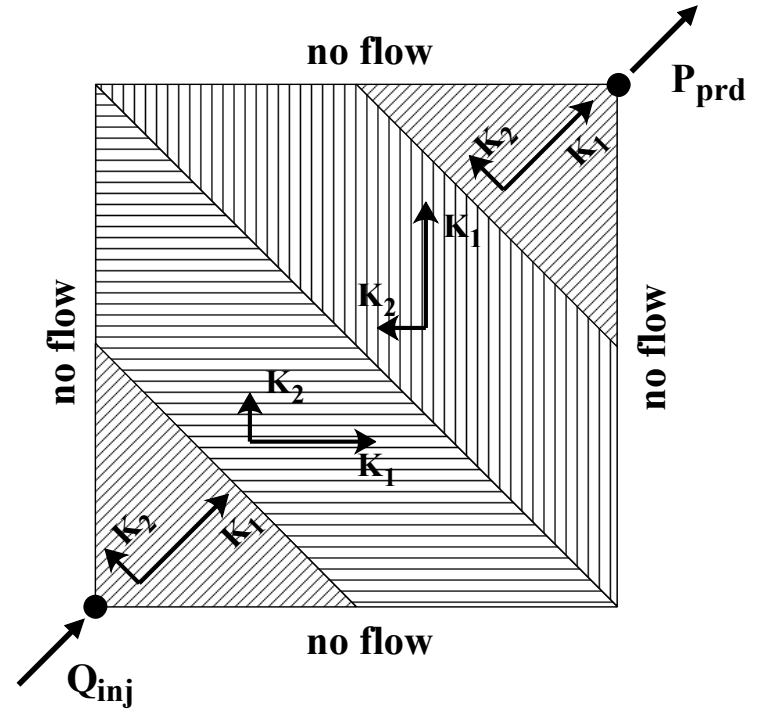

(a) Geometry and permeability field

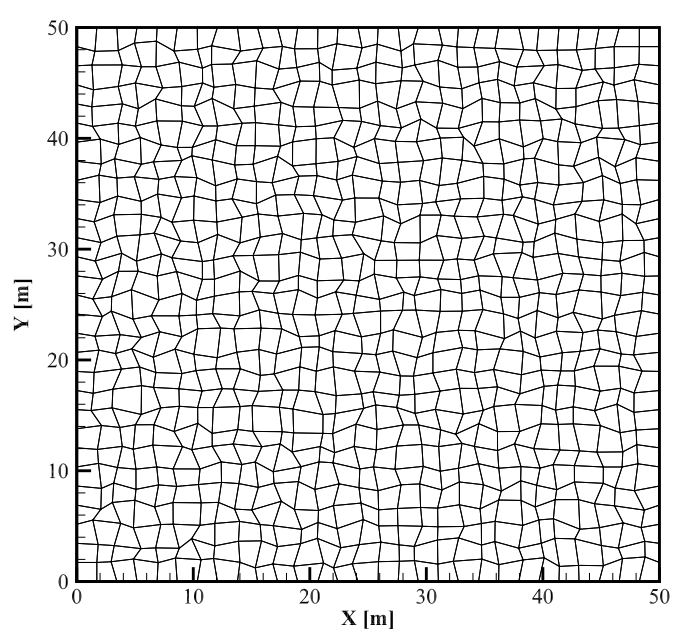

(b) Quadrilateral grid

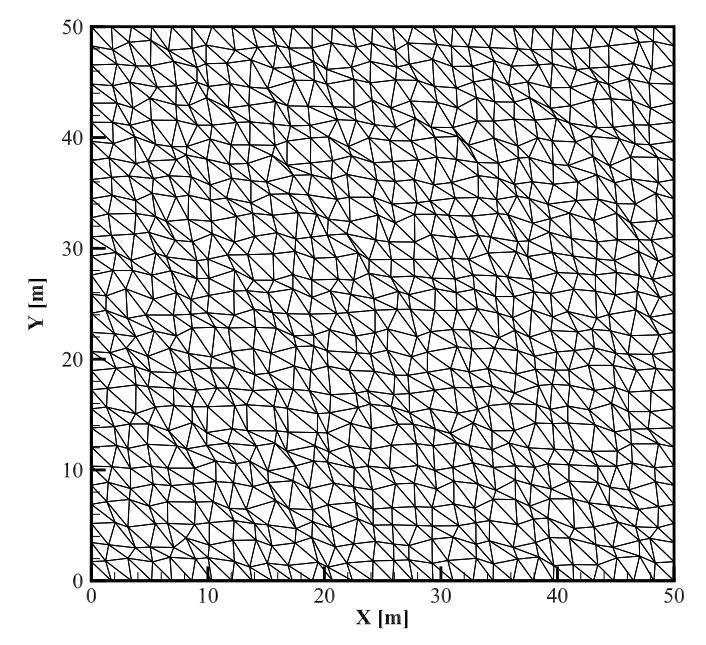

(c) Triangular grid

Figure 9: Unstructured grids used in Result section

\subsection{Evaluation of Procedures for Solving Pressure Equation}

To investigate different numerical methods for solving pressure equation, a test case was designed in which a quarter of the five-spot problem is solved in a heterogeneous anisotropic domain. Anisotropy ratio is defined as $\frac{K_{1}}{K_{2}}$ and the permeability tensor is rotated in various parts of the domain. Figure 9a shows geometry and permeability variations in the domain. Reservoir is initially filled with a mixture of $\mathrm{C}_{2}$ and $\mathrm{C}_{3}$ in liquid phase while $\mathrm{C}_{1}$ in vapor phase is injected from bottom left corner and liquid is produced from top right corner. Fluid properties and other parameters are given in Table 5. Elliptic part of the problem is solved using CVDTPS, CVDFPS, and CVDFE methods in unstructured quadrilateral and triangular grids of Figures $9 \mathrm{~b}$ and $9 \mathrm{c}$, respectively. ROE scheme is used for solving hyperbolic system of equations in all cases while CFL is set to 0.5. The grids are constructed from a uniform structured Cartesian mesh by applying random deformation of the order of 0.3 of cell size. In both quadrilateral and triangular cases, the deformation parameter is set to a large number in order to generate highly distorted grids that make the comparison between different pressure schemes possible in difficult situations.

Figures 10a to 10 show the results of $\mathrm{C}_{1}$ mole fraction at 0.50 Pore Volume Injection (PVI) 


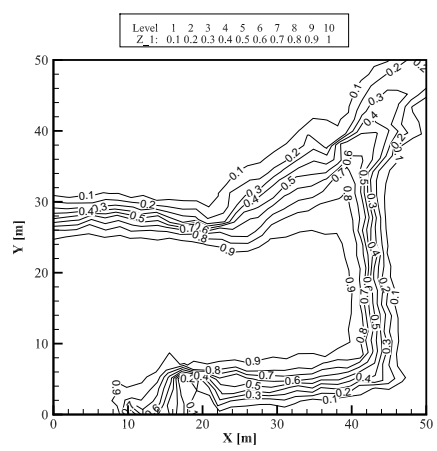

(a) TPS at $\frac{K_{1}}{K_{2}}=\frac{1000}{100} \frac{\mathrm{mD}}{\mathrm{mD}}$

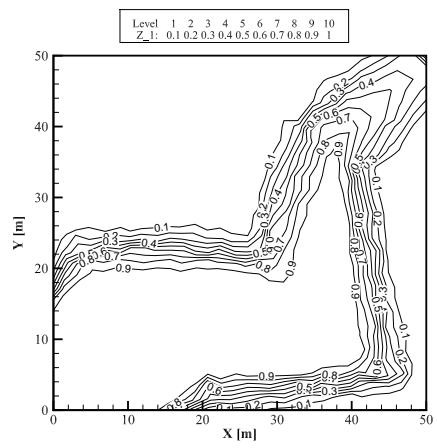

(d) FPS at $\frac{K_{1}}{K_{2}}=\frac{1000}{50} \frac{\mathrm{mD}}{\mathrm{mD}}$

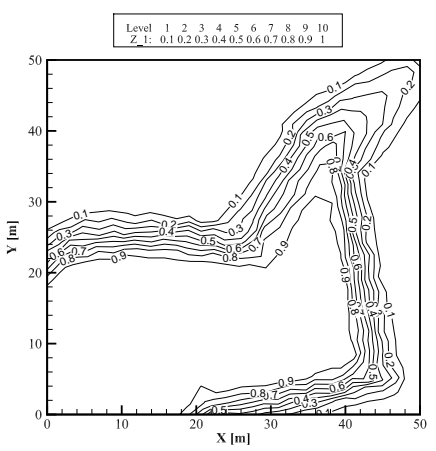

(b) FPS at $\frac{K_{1}}{K_{2}}=\frac{1000}{100} \frac{\mathrm{mD}}{\mathrm{mD}}$

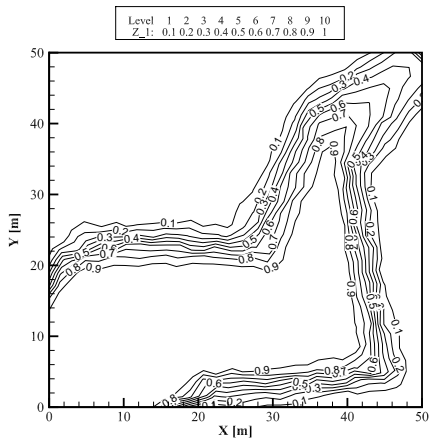

(e) CVDFE at $\frac{K_{1}}{K_{2}}=\frac{1000}{50} \frac{\mathrm{mD}}{\mathrm{mD}}$

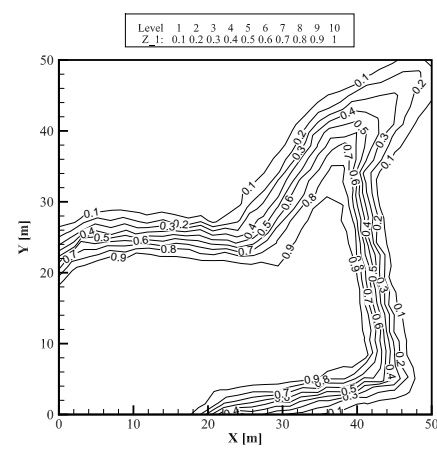

(c) CVDFE at $\frac{K_{1}}{K_{2}}=\frac{1000}{100} \frac{\mathrm{mD}}{\mathrm{mD}}$

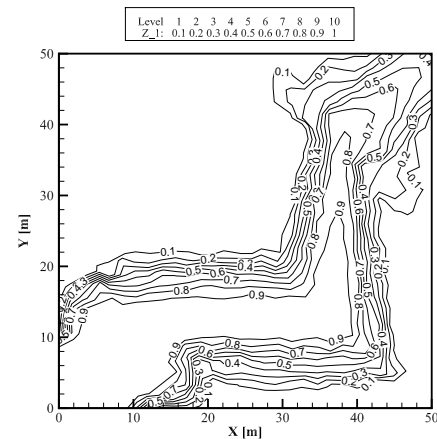

(f) CVDFE at $\frac{K_{1}}{K_{2}}=\frac{1000}{10} \frac{\mathrm{mD}}{\mathrm{mD}}$

Figure 10: Result of $\mathrm{C}_{1}$ mole fraction in second test case at 0.50 PVI using a quadrilateral grid for various $\frac{K_{1}}{K_{2}}$.

using various pressure schemes on the quadrilateral grid of Figure 9b. In Figures 10a, 10b, and 10c anisotropy ratio is $\frac{K_{1}}{K_{2}}=\frac{1000}{100} \frac{\mathrm{mD}}{\mathrm{mD}}$ and it can be seen from these figures that all three methods produce somewhat similar results in quadrilateral grid. In Figures 10d and 10e, anisotropy ratio increases to $\frac{K_{1}}{K_{2}}=\frac{1000}{50} \frac{\mathrm{mD}}{\mathrm{mD}}$ for which FPS and CVDFE methods produces similar results while TPS diverges and fails to predict a physical solution. If anisotropy ratio rises to $\frac{K_{1}}{K_{2}}=\frac{1000}{10} \frac{\mathrm{mD}}{\mathrm{mD}}$ as depicted in Figure 10f, only CVDFE method produces an acceptable solution, while both TPS and FPS methods diverge at the early time steps.

Similar results can be obtained using triangular grid of Figure 9c as depicted in Figures 11a to 11e. Since the latter grid is more complicated than its quadrilateral counterpart, it can be seen that only FPS and CVDFE are able to produce acceptable solutions for $\frac{K_{1}}{K_{2}}=\frac{1000}{100} \frac{\mathrm{mD}}{\mathrm{mD}}$ and $\frac{K_{1}}{K_{2}}=\frac{1000}{50} \frac{\mathrm{mD}}{\mathrm{mD}}$ cases albeit with higher numerical dissipation compared to the result of quadrilateral grid as shown in Figures 10. Finally, as shown in Figure 11e, only the CVDFE produces an acceptable solution when anisotropy ratio rises to $\frac{K_{1}}{K_{2}}=\frac{1000}{10} \frac{\mathrm{mD}}{\mathrm{mD}}$. It should be noted that all methods (TPS, FPS, and CVDFE) produce reasonable solutions for anisotropy ratios up to $\frac{K_{1}}{K_{2}}=\frac{1000}{100} \frac{\mathrm{mD}}{\mathrm{mD}}$ but are not shown here for the sake of conciseness.

It can be seen that, while FPS (and TPS in simpler cases) does not produce cross-flow dissipation (spread the flow information along permeability discontinuities), however, they face difficulties dealing with highly distorted grids with high anisotropy ratios. On the other hand, CVDFEM can be used with much lower quality grids and heterogeneous permeability fields albeit by generating some dissipation where high contrast discontinuities occur as can be seen in Figure 10f, near permeability discontinuities. This phenomenon originates from FE averaging of flow variables in CVFEM not from averaging rock properties as suggested here in CVDFEM. However, as it can be seen from the results of this section, CVDFE solutions can compete well in all cases, and dissipations only occurs in very high anisotropy ratios for which other methods fail to converge to a physical solution. 


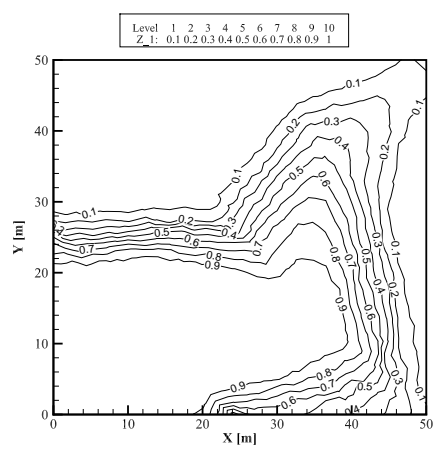

(a) FPS at $\frac{K_{1}}{K_{2}}=\frac{1000}{100} \frac{\mathrm{mD}}{\mathrm{mD}}$

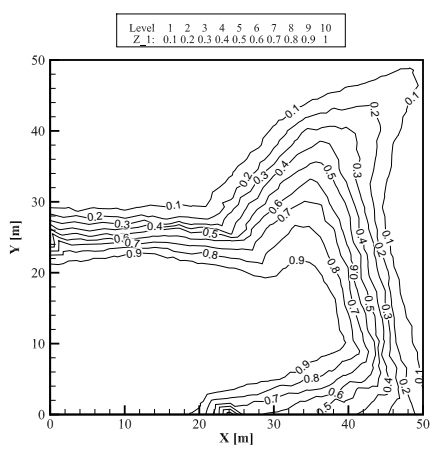

(b) CVDFE at $\frac{K_{1}}{K_{2}}=\frac{1000}{100} \frac{\mathrm{mD}}{\mathrm{mD}}$

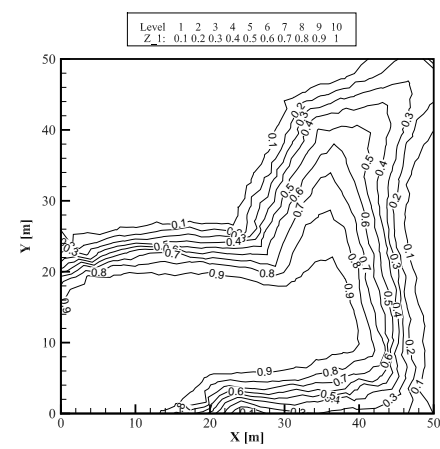

(c) FPS at $\frac{K_{1}}{K_{2}}=\frac{1000}{50} \frac{\mathrm{mD}}{\mathrm{mD}}$

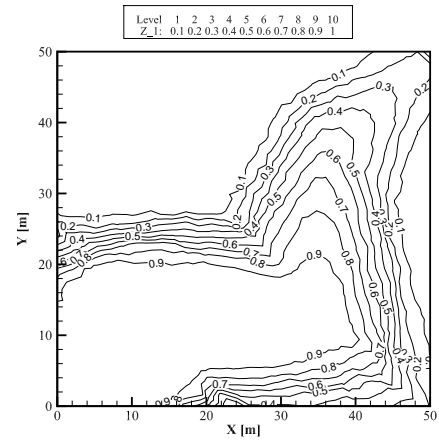

(d) $\mathrm{CVDFE} \frac{K_{1}}{K_{2}}=\frac{1000}{50} \frac{\mathrm{mD}}{\mathrm{mD}}$

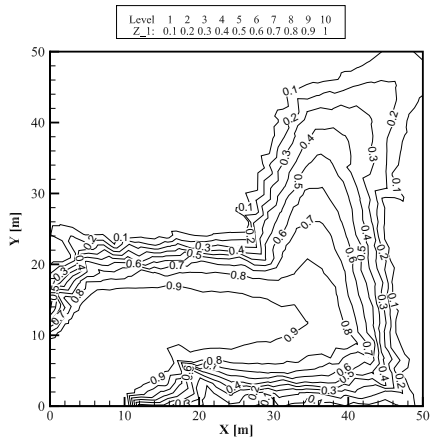

(e) CVDFE at $\frac{K_{1}}{K_{2}}=\frac{1000}{10} \frac{\mathrm{mD}}{\mathrm{mD}}$

Figure 11: Result of $\mathrm{C}_{1}$ mole fraction in second test case at $0.50 \mathrm{PVI}$ using a triangular grid for various $\frac{K_{1}}{K_{2}}$.

\subsection{Evaluation of Schemes for Solving Mass Conservation Equations}

In this section, the performance of numerical schemes (ROE, HLL, LLF, and MDW) used for solving the mass conservation equations are assessed. A highly heterogeneous and anisotropic reservoir is considered and CVDFEM is used for solving the pressure equation. The geometry of problem, location of wells, and rock properties are the same as the second test case; however, here the problem involves flow of a five-component two-phase fluid. Fluid properties are given in Table 6. In this test case, $\mathrm{C}_{1}$ in vapor phase is injected from bottom left corner into a reservoir initially contains $\mathrm{C}_{2}, \mathrm{nC}_{4}, \mathrm{nC}_{10}$, and $\mathrm{nC}_{16}$ components in the liquid phase.

Figure 12 shows the results of vapor saturation at 0.5 PVI using various first order numerical schemes and quadrilateral grid of Figure $9 \mathrm{~b}$. CFL is set to 0.5 as before. The reason for using vapor saturation for the comparison is that this parameter is a secondary one which is obtained from a combination all mole fractions and pressure, hence it can be an indication of overall quality of solution. Moreover, vapor saturation is a tangible physical properties of the flow in porous media. It is evident from this figure that the MDW and HLL schemes produce superior results. The ROE scheme shows abnormal behavior near the production well where pressure gradient and velocities are higher. Ideal route of flow is shown in Figure 13a, which is composed of four straight line segments connecting points with various permeability tensor. Figure $13 \mathrm{~b}$ shows the result of vapor saturation along the route shown in Figure 13a. Figure 13b clearly shows the features of flow such as shocks, discontinuities, and rarefactions. Figure 13c shows similar result for the triangular grid (Figure 9c). The superiority of MDW and HLL schemes can be clearly seen from Figure 13b and Figure 13c.

To investigate the effect of high-order extension procedures presented in this paper, results of MUSCL and WENO reconstructions are shown in this section. Figure 14 shows the results of vapor saturation using high-order spatial reconstruction. In Figure 14 MDW scheme is used as the base method. It is evident from this figure that the streamline diffusion decreased greatly using either MUSCL or WENO high-order methods. It can be seen from Figure 14 that WENO and 


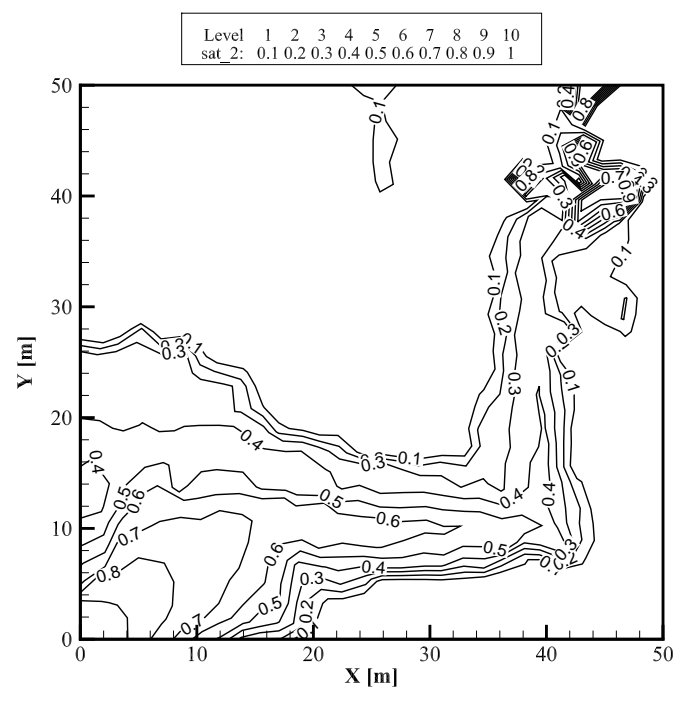

(a) ROE

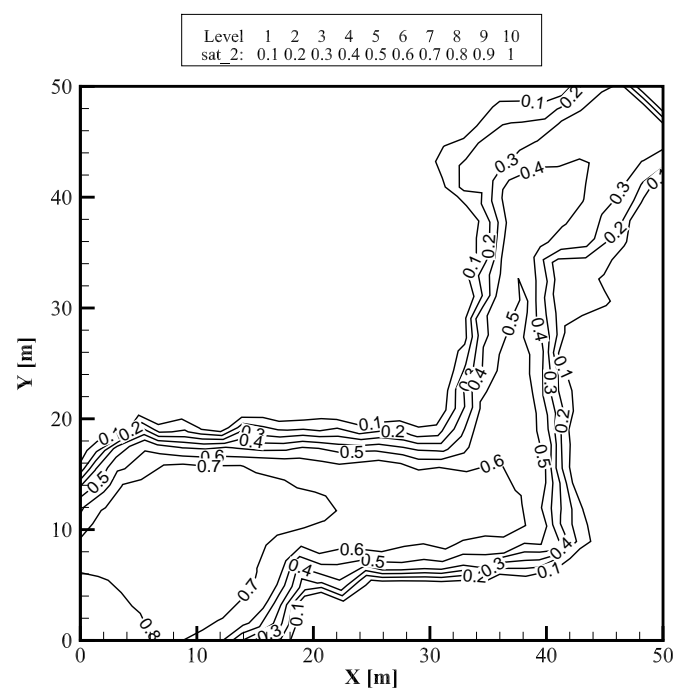

(c) LLF

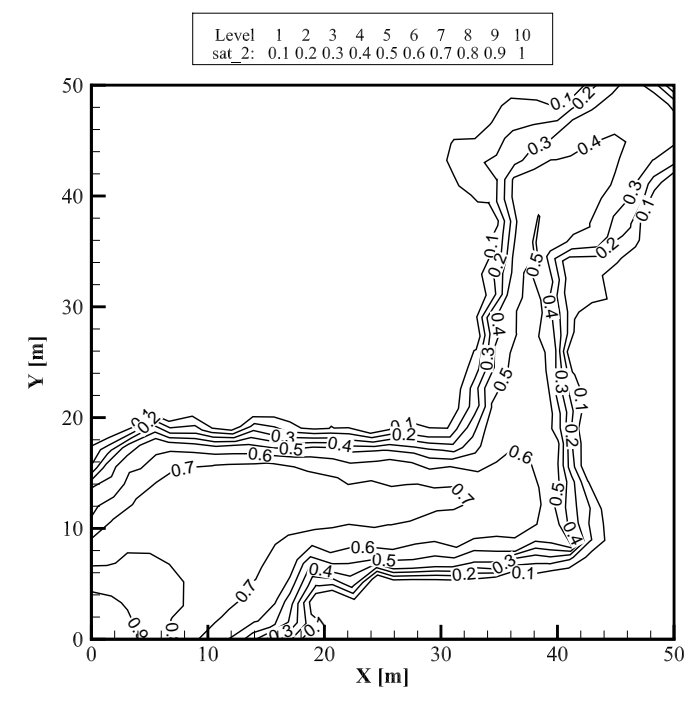

(b) MDW

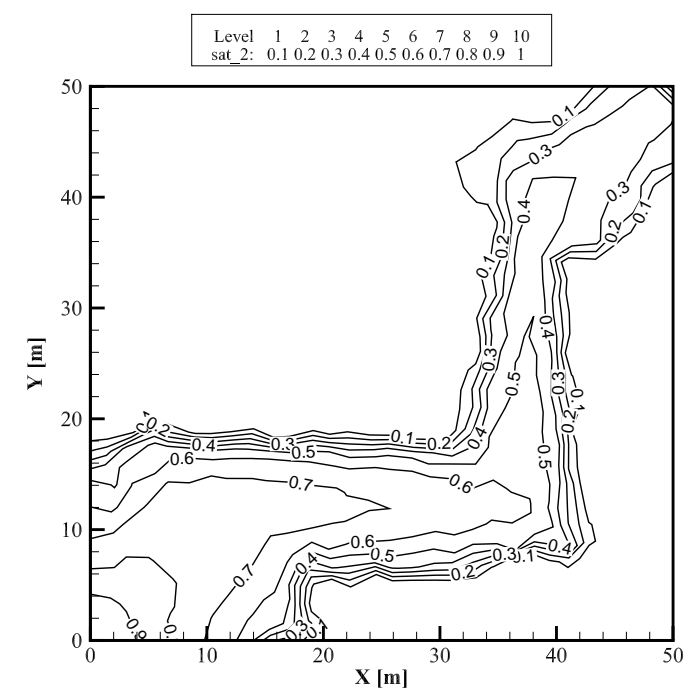

(d) HLL

Figure 12: Result of vapor saturation in third test case at 0.50 PVI using a quadrilateral grid and various hyperbolic schemes 


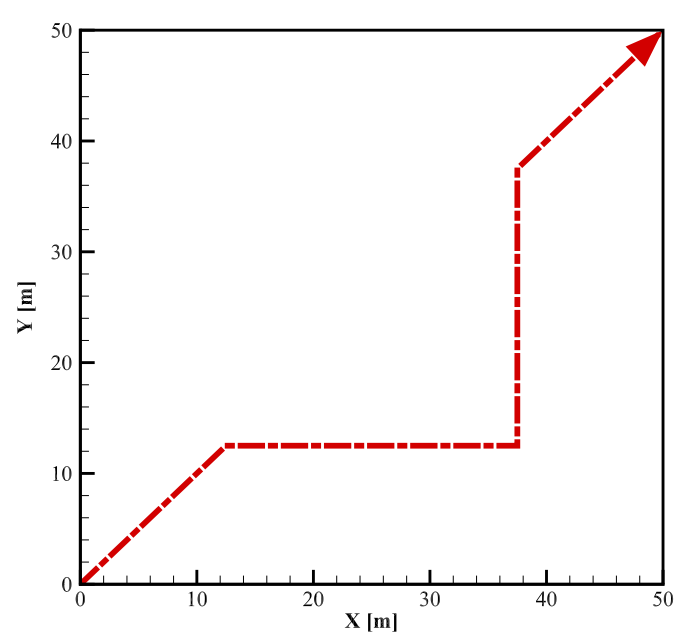

(a) route of flow

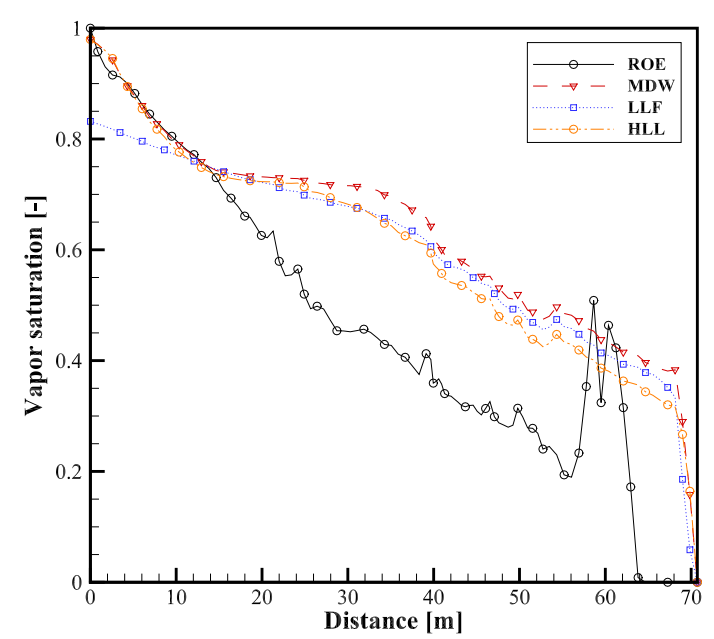

(b) solution on quadrilateral grid

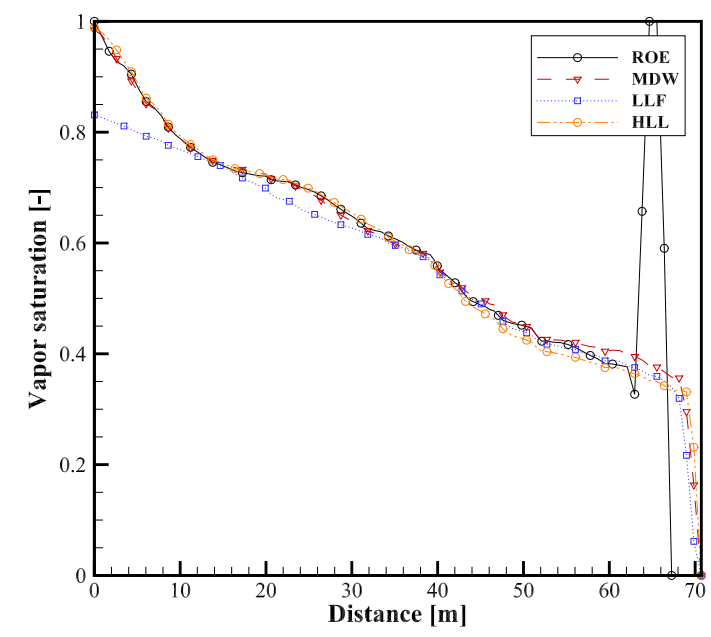

(c) solution on triangular grid

Figure 13: Result of vapor saturation in third test case at 0.50 PVI using various hyperbolic schemes 


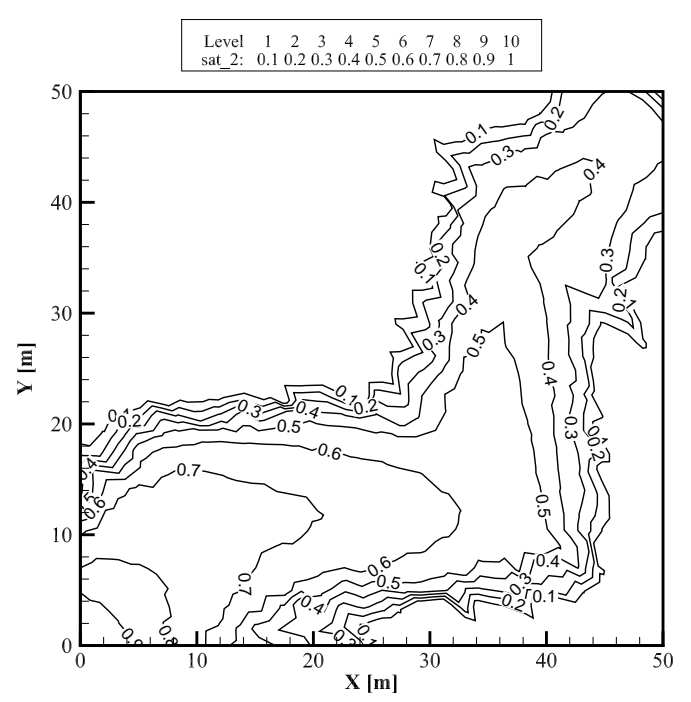

(a) First order

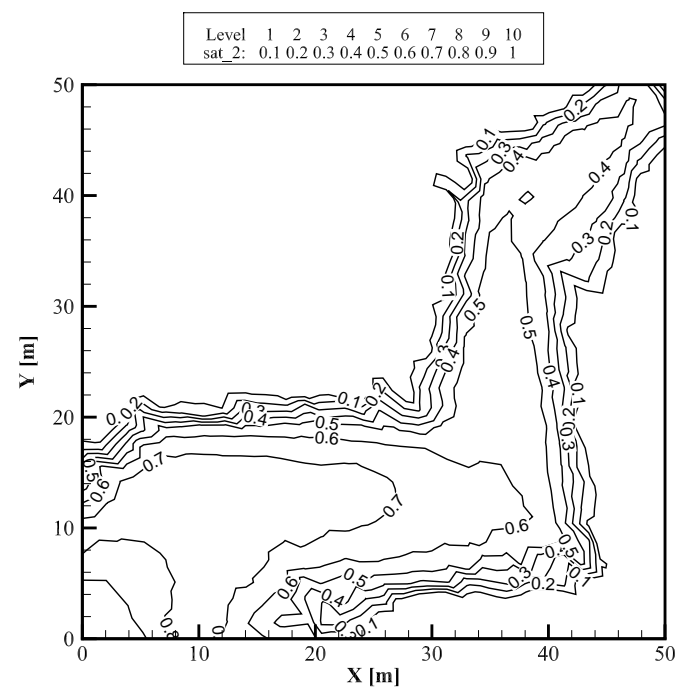

(c) WENO

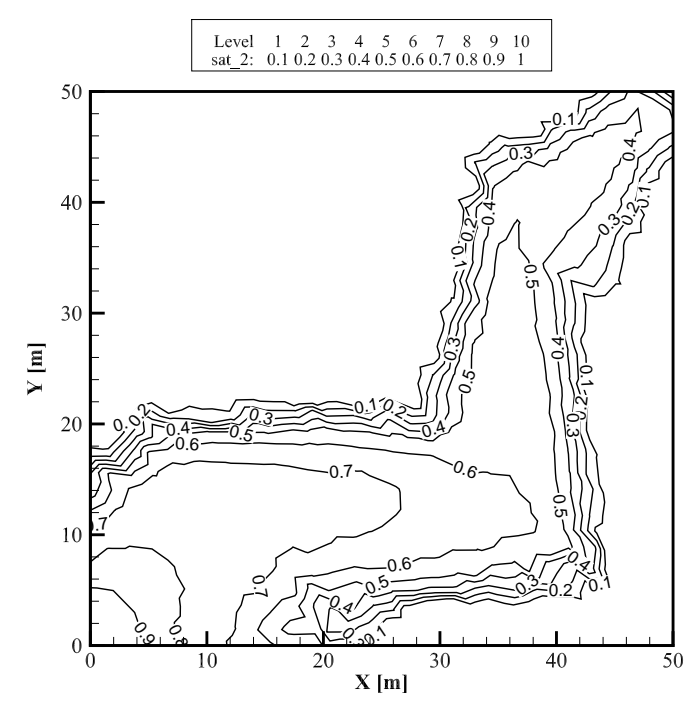

(b) MUSCL

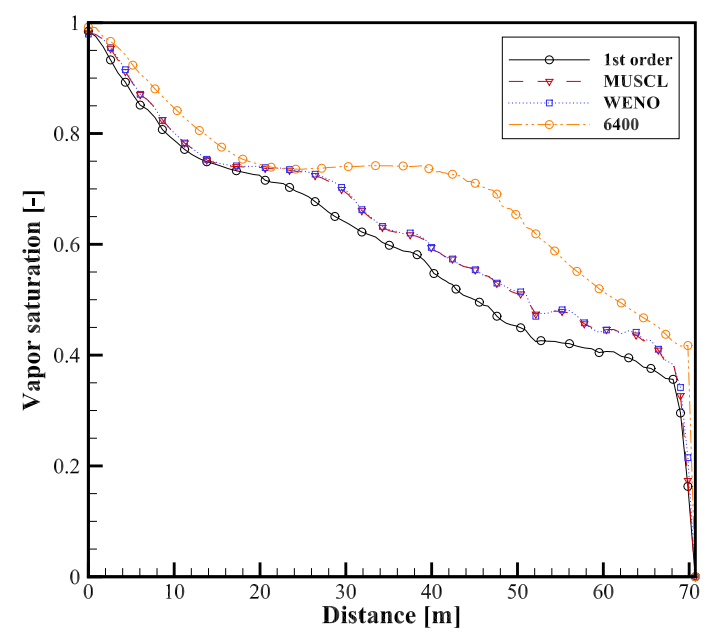

(d) solution on flow route

Figure 14: Result of vapor saturation in third test case at 0.50 PVI using high-order reconstructions

MUSCL reconstructions produce similar results. In fact, authors' experiences indicate no significant preference between MUSCL and WENO reconstructions for compositional flow in heterogeneous anisotropic porous media. Figure 14d shows the result of vapor saturation along the flow route. It can be seen from this figure that, flow features were captured with greater resolution when high-order schemes are used.

Figure 15 shows the effect of grid refinement for the current problem on the consecutively refined quadrilateral grids. As expected, the numerical diffusion decreases as the number of cells increases. Figure 15d shows the result of vapor saturation along the route shown in Figure 13a. It is evident that by increasing the number of cells, the accuracy of solution of conservation equation increases.

Computational run times (seconds) of various schemes are compared in Figure 16a as a function of the number of nodes. This figure shows that MDW scheme has the lowest computational cost, while the LLF has the highest. In fact, as the number of chemical components increases in the compositional simulation, the gap between the run time of the MDW and that of other schemes increases.

From a theoretical point of view, the computational costs of solving hyperbolic equations in 


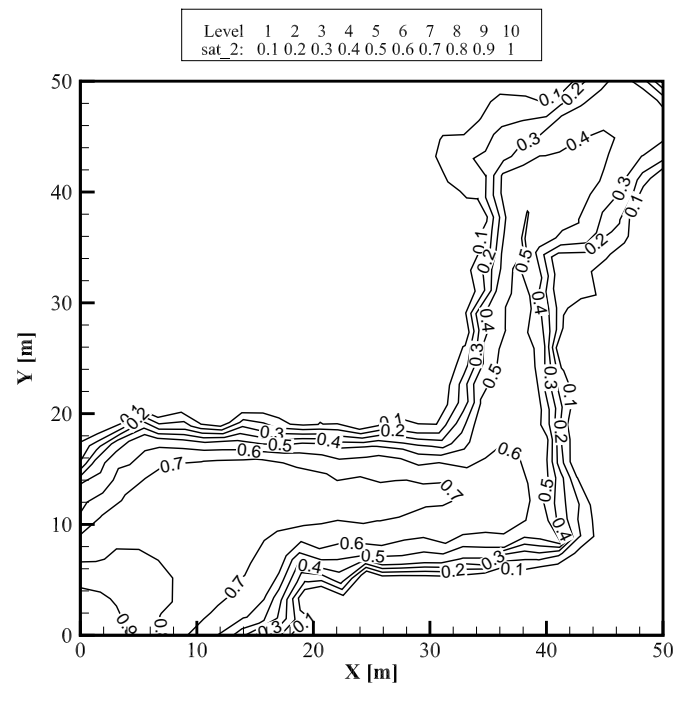

(a) 900 cells

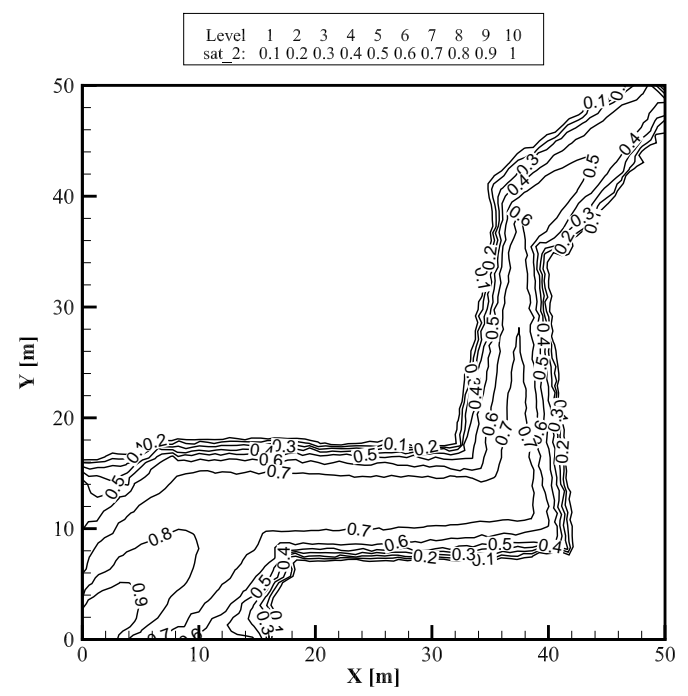

(c) 6400 cells

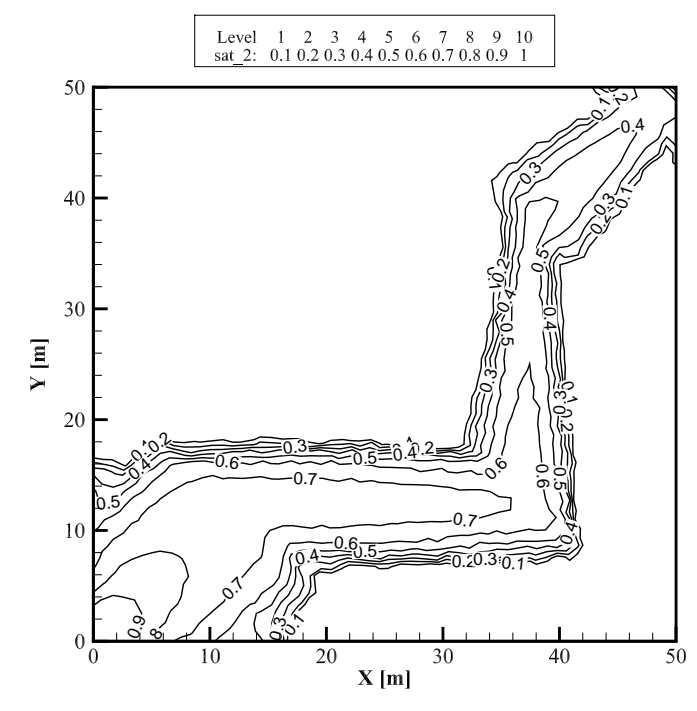

(b) 3600 cells

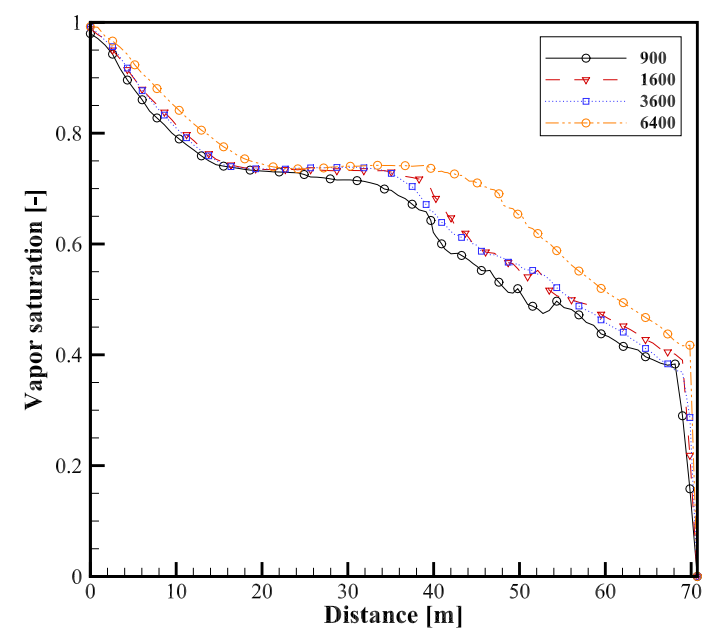

(d) solution on flow route

Figure 15: Result of vapor saturation in third test case at 0.50 PVI on consequently fined grids 

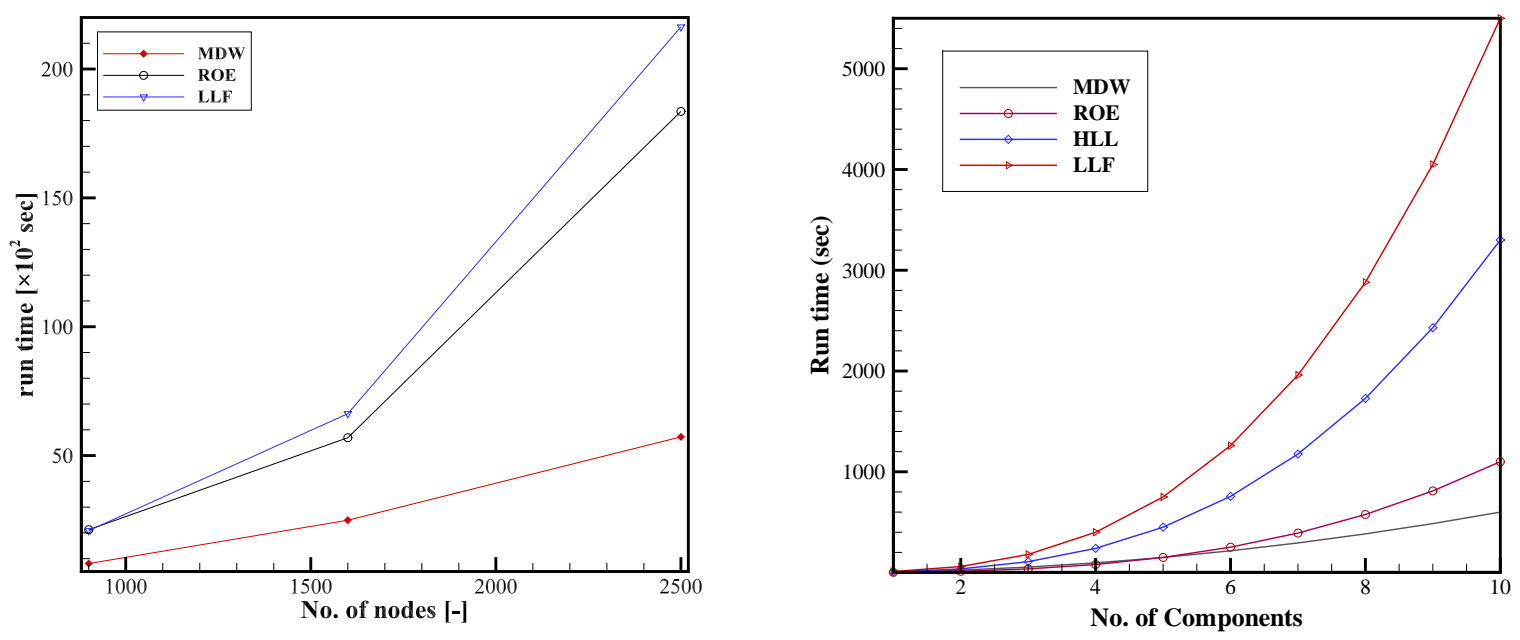

(a) Computational cost with respect to number of nodes (b) Theoretical computational cost for each flux calculation

Figure 16: Computational cost of hyperbolic schemes

the compositional simulation depends linearly on the costs of flux approximation. The latter cost may be divided into two categories: 1) the cost of fluid properties calculation including: stability analysis and flash procedure, 2) the cost of linear algebra and matrices computations, including inversion, multiplications, and eigen-structure approximation used for flux calculations. According to the authors experiences in compositional simulation, the computational resources needed for calculation of fluid properties surpasses other costs by far, especially when fluid systems with several components are to be simulated. Table 3 shows the the number of times fluid properties must be calculated for approximating numerical fluxes in each scheme at each time step. Figure 16b shows theoretical computational cost. Figure 16b suggests that the MDW outperforms other schemes from computational cost point of view and the superiority of MDW is more prominent as the number of chemical components increases.

Table 3: Number of times fluid properties must be calculated for flux approximation at each time step

\begin{tabular}{|c|c|c|c|c|}
\hline Scheme & MDW & ROE & HLL & LLF \\
\hline Number of calculations & 10 & $n_{c}+1$ & $3\left(n_{c}+1\right)$ & $5\left(n_{c}+1\right)$ \\
\hline
\end{tabular}

Table 4: Problem properties for first test case

\begin{tabular}{|c|c|c|c|}
\hline Quantity & Unit & \multicolumn{2}{|c|}{ Value } \\
\hline Components & - & $\mathrm{C}_{1}$ & $\mathrm{nC}_{10}$ \\
\hline Injection mole fraction & - & 1.00 & 0.00 \\
\hline Initial mole fraction & - & 0.00 & 1.00 \\
\hline Initial pressure & $\mathrm{psia}$ & 1500 \\
\hline Temperature & $\mathrm{F}$ & 159.4 \\
\hline Injection rate at standard condition & $\mathrm{ft}^{3} /$ day & 100,000 \\
\hline BHP of production well & $\mathrm{psia}$ & 1500 \\
\hline Porosity & - & 0.2 \\
\hline Relative permeability & - & \multicolumn{2}{|c|}{$k_{r}^{\alpha}=\left(s^{\alpha}\right)^{2}$} \\
\hline
\end{tabular}


Table 5: Problem properties for second test case

\begin{tabular}{|c|c|c|c|c|}
\hline Quantity & Unit & \multicolumn{3}{|c|}{ Value } \\
\hline Components & - & $\mathrm{C}_{1}$ & $\mathrm{C}_{2}$ & $\mathrm{C}_{3}$ \\
\hline Injection mole fraction & - & 0.90 & 0.10 & 0.00 \\
\hline Initial mole fraction & - & 0.00 & 0.25 & 0.75 \\
\hline Initial pressure & $\mathrm{MPa}$ & \multicolumn{3}{|c|}{6.9} \\
\hline Temperature & $\mathrm{K}$ & \multicolumn{3}{|c|}{311} \\
\hline Injection rate & $\mathrm{m}^{3} /$ day & \multicolumn{3}{|c|}{0.017} \\
\hline Production pressure & $\mathrm{MPa}$ & \multicolumn{3}{|c|}{6.9} \\
\hline Porosity & - & \multicolumn{3}{|c|}{0.2} \\
\hline Relative permeability & - & \multicolumn{3}{|c|}{$k_{r}^{\alpha}=s^{\alpha}$} \\
\hline
\end{tabular}

Table 6: Problem properties for third test case

\begin{tabular}{|c|c|c|c|c|c|c|}
\hline Quantity & Unit & \multicolumn{5}{|c|}{ Value } \\
\hline Components & - & $\mathrm{C}_{1}$ & $\mathrm{C}_{2}$ & $\mathrm{nC}_{4}$ & $\mathrm{nC}_{10}$ & $\mathrm{nC}_{16}$ \\
\hline Injection mole fraction & - & 1.00 & 0.00 & 0.00 & 0.00 & 0.00 \\
\hline Initial mole fraction & - & 0.00 & 0.25 & 0.25 & 0.25 & 0.25 \\
\hline Initial pressure & $\mathrm{MPa}$ & \multicolumn{5}{c|}{6.9} \\
\hline Temperature & $\mathrm{K}$ & \multicolumn{5}{c|}{0.017} \\
\hline Injection rate & $\mathrm{m}^{3} /$ day & \multicolumn{5}{c|}{6.9} \\
\hline Production pressure & $\mathrm{MPa}$ & \multicolumn{5}{c|}{0.2} \\
\hline Porosity & - & \multicolumn{5}{c|}{$k_{r}^{\alpha}=\left(s^{\alpha}\right)^{2}$} \\
\hline Relative permeability & - & \multicolumn{5}{c}{} \\
\hline
\end{tabular}

Table 7: PVT properties of components [50]

\begin{tabular}{|c|c|c|c|c|c|c|}
\hline & & $T_{c}$ & $p_{c}$ & $v_{c}$ & $M_{w}$ & $\omega$ \\
\cline { 3 - 7 } Name & Symbol & {$[\mathrm{K}]$} & {$[\mathrm{MPa}]$} & {$\left[\mathrm{m}^{3} / \mathrm{kg}-\mathrm{mol}\right]$} & {$[\mathrm{kg} / \mathrm{kg}-\mathrm{mol}]$} & {$[-]$} \\
\hline Methane & $\mathrm{C}_{1}$ & 190.56 & 4.599 & 0.0986 & 16.043 & 0.0115 \\
\hline Ethane & $\mathrm{C}_{2}$ & 305.32 & 4.872 & 0.1455 & 30.070 & 0.0995 \\
\hline Propane & $\mathrm{C}_{3}$ & 369.83 & 4.248 & 0.2000 & 44.096 & 0.1523 \\
\hline n-Butane & $\mathrm{nC}_{4}$ & 425.12 & 3.796 & 0.2550 & 58.123 & 0.2002 \\
\hline n-Decane & $\mathrm{nC}_{10}$ & 617.70 & 2.110 & 0.6000 & 142.285 & 0.4923 \\
\hline n-Hexadecane & $\mathrm{nC}_{16}$ & 723.00 & 1.400 & 0.9440 & 226.446 & 0.7174 \\
\hline
\end{tabular}

\section{Conclusion}

In this paper, a new method was introduced for solving pressure equation in full-tensor anisotropic and heterogeneous porous media. This method which is called CVDFE belongs to the CVD framework and was shown to be able to simulate multi-phase compositional flow in highly anisotropic and heterogeneous porous media.

It was shown that, CVDFE method can be preferred choice when grid does not comply with the principal directions of permeability field or in cases of highly heterogeneous permeability field with distorted grids. However, despite its stability and convergence preferences on heterogeneous permeability fields, CVDFE method suffers from the so-called spread of flow information when crossing sudden permeability variations. It should be noted that, this occurs in cases with very high anisotropy ratio where all other methods fail to produce physical solutions.

Several numerical schemes were used to solve hyperbolic system of mass conservation equations including ROE, MDW, LLF, and HLL. Theses schemes have been previously tested by the authors for one-dimensional problems and in this paper they are extended to two-dimensional unstructured grids. 
The results suggest that MDW scheme outperforms other schemes studied in this paper from both accuracy and computational cost points of view.

The proposed high-order extensions which are based on one-dimensional reconstruction was shown to improve the accuracy of solution.

\section{Nomenclature}

$n_{c} \quad$ number of components, [-]

$n_{p} \quad$ number of phases, [-]

$s^{\alpha} \quad$ saturation of phase $\alpha,[-]$

$\mu^{\alpha} \quad$ viscosity of phase $\alpha,\left[\mathrm{ML}^{-1} \mathrm{~T}^{-1}\right]$

$k_{r}^{\alpha} \quad$ relative permeability of phase $\alpha,[-]$

$\rho^{\alpha} \quad$ molar density of phase $\alpha,\left[\mathrm{NL}^{-3}\right]$

$\varrho^{\alpha} \quad$ mass density of phase $\alpha,\left[\mathrm{ML}^{-3}\right]$

$\lambda^{\alpha} \quad$ mobility of phase $\alpha,\left[\mathrm{M}^{-1} \mathrm{LT}\right]$

$Z^{\alpha} \quad$ compressibility of phase $\alpha,[-]$

$\beta_{v} \quad$ mole fraction of vapor phase, [-]

$p \quad$ pressure, $\left[\mathrm{ML}^{-1} \mathrm{~T}^{-2}\right]$

$T$ temperature, $[\Theta]$

$z_{i} \quad$ mole fraction of component $i,[-]$

$K_{i} \quad$ equilibrium ratio of component $i,[-]$

$f_{i}^{\alpha} \quad$ fugacity of component $i$ in phase $\alpha$, $\left[\mathrm{ML}^{-1} \mathrm{~T}^{-2}\right]$

$x_{i}^{\alpha} \quad$ mole fraction of component $i$ in phase $\alpha$, $[-]$

m vector of component mole numbers, $[\mathrm{N}]$

$\mathbf{u}^{\alpha} \quad$ velocity of phase $\alpha,\left[\mathrm{LT}^{-1}\right]$

$\mathbf{u}_{i} \quad$ vector of velocity for component $i,\left[\mathrm{LT}^{-1}\right]$

$\nu_{i}^{\text {tot }}$ total partial molar volume of $i$ th component, $\left[\mathrm{N}^{-1} \mathrm{~L}^{3}\right]$

$c_{f} \quad$ fluid compressibility, $\left[\mathrm{M}^{-1} \mathrm{LT}^{2}\right]$

$c_{r} \quad$ rock compressibility, $\left[\mathrm{M}^{-1} \mathrm{LT}^{2}\right]$

$\mathbf{K}$ tensor of absolute permeability, $\left[\mathrm{L}^{2}\right]$

$\phi \quad$ porosity, [-]

$t \quad$ time, $[\mathrm{T}]$

$\Delta t \quad$ time step size, $[\mathrm{T}]$
$V_{j} \quad$ volume of $j$ th $\mathrm{CV},\left[\mathrm{L}^{3}\right]$

$x \quad$ length coordinate, $[\mathrm{L}]$

$y \quad$ width coordinate, $[\mathrm{L}]$

$F \quad$ Elliptic flux, $\left[\mathrm{LT}^{-1}\right]$

f vector of component hyperbolic fluxes, $\left[\mathrm{NLT}^{-1}\right]$

$\hat{\mathbf{f}} \quad$ vector of component numerical fluxes, $\left[\mathrm{NLT}^{-1}\right]$

Q vector of component molar sources/sinks, $\left[\mathrm{NT}^{-1}\right]$

$\lambda \quad$ numerical wave speed, $\left[\mathrm{LT}^{-1}\right]$

$\lambda_{j} \quad j$ th eigen-value of Jacobian matrix, $\left[\mathrm{LT}^{-1}\right]$

$\boldsymbol{\Lambda}$ diagonal matrix of eigenvalues, $\left[\mathrm{LT}^{-1}\right]$

$\mathbf{r}_{j} \quad j$ th eigen-vector of Jacobian matrix, [-]

$\mathbf{R}$ matrix of eigenvectors of Jacobian matrix, $\left[\mathrm{LT}^{-1}\right]$

$\varphi \quad$ slope limiter, [-]

CFL Courant-Friedrichs-Lewy number, [-]

HLL Harten-Lax-van Leer scheme

ROE Roe-based scheme

LLF Local Lax- Friedrichs scheme

MDW Modified Dominant Wave scheme

$\mathbf{A}_{L}, \mathbf{A}_{R}$ coeficient matrix, [-]

$\mathbf{B}_{L}, \mathbf{B}_{R}$ coeficient matrix, [-]

C coeficient matrix, [-]

D coeficient matrix, [-]

$N \quad$ shape function, [-]

$n_{E} \quad$ number of edges of CV, [-]

$n_{V} \quad$ number of vertices of cell, [-]

$n_{C V}$ number of $\mathrm{CV},[-]$

r position vector, $[\mathrm{L}]$

$\xi \quad$ local coordinate of cell, [-] 
$\eta \quad$ local coordinate of cell, [-]

\section{subscripts}

$i \quad$ component index

$\alpha \quad$ phase index

$l \quad$ liquid phase

$v \quad$ vapor phase

j node (CV) index

e $\quad$ edge index
$L \quad$ left state

M mean state

$R \quad$ right state

$S, E, N, W$ integration points

$s, e, n, w, m$ mid-points of cell

$I, J \quad$ extended point index

$M \quad$ mid-point index

$V \quad$ vertex index

\section{References}

[1] M. Pal, M. G. Edwards, A family of multi-point flux approximation schemes for general element types in two and three dimensions with convergence performance, International Journal for Numerical Methods in Fluids 69 (2012) 1797-1817.

[2] I. Aavatsmark, T. Barkve, O. Bœ, T. Mannseth, Discretization on Unstructured Grids for Inhomogeneous, Anisotropic Media. Part I: Derivation of the Methods, SIAM Journal on Scientific Computing 19 (5) (1998) 1700-1716. doi:10.1137/S1064827595293582. URL http://epubs.siam.org/doi/abs/10.1137/S1064827595293582

[3] M. G. Edwards, C. F. Rogers, Finite volume discretization with imposed flux continuity for the general tensor pressure equation, Computational Geosciences 2 (4) (1998) 259-290.

[4] R. A. Klausen, T. F. Russell, Relationships among some locally conservative discretization methods which handle discontinuous coefficients, Computational Geosciences 8 (4) (2005) 341377. doi:10.1007/s10596-005-1815-9.

URL http://link.springer.com/10.1007/s10596-005-1815-9

[5] H. A. Friis, M. G. Edwards, A family of MPFA finite-volume schemes with full pressure support for the general tensor pressure equation on cell-centered triangular grids, Journal of Computational Physics 230 (1) (2011) 205-231. doi:10.1016/j.jcp.2010.09.012.

URL http://dx.doi.org/10.1016/j.jcp.2010.09.012http://linkinghub.elsevier. com/retrieve/pii/S0021999110005061

[6] K. Aziz, A. Settari, Petroleum Reservoir Simulation, Applied Science Publication Ltd., London, UK, 1979.

[7] M. G. Edwards, Unstructured, control-volume distributed, full-tensor finite-volume schemes with flow based grids, Computational Geosciences 6 (3) (2002) 433-452.

[8] I. Aavatsmark, An introduction to multipoint flux approximations for quadrilateral grids, Computational Geosciences 6 (3) (2002) 405-432.

[9] I. Aavatsmark, G. T. Eigestad, R. A. Klausen, M. F. Wheeler, I. Yotov, Convergence of a symmetric MPFA method on quadrilateral grids, Computational Geosciences 11 (4) (2007) 333-345. doi:10.1007/s10596-007-9056-8.

URL http://link.springer.com/10.1007/s10596-007-9056-8

[10] M. G. Edwards, H. Zheng, Double-families of quasi-positive Darcy-flux approximations with highly anisotropic tensors on structured and unstructured grids, Journal of Computational Physics 229 (3) (2010) 594-625. doi:10.1016/j.jcp.2009.09.037.

URL http://linkinghub.elsevier.com/retrieve/pii/S0021999109005294 
[11] M. G. Edwards, Higher-resolution hyperbolic-coupled-elliptic flux-continuous CVD schemes on structured and unstructured grids in 2-D, International Journal for Numerical Methods in Fluids 51 (9-10) (2006) 1059-1077.

[12] M. G. Edwards, H. Zheng, Quasi M-matrix multifamily continuous Darcy-flux approximations with full pressure support on structured and unstructured grids in three dimensions, SIAM Journal on Scientific Computing 33 (2) (2011) 455-487.

[13] S. Lamine, M. G. Edwards, Higher order multidimensional upwind convection schemes for flow in porous media on structured and unstructured quadrilateral grids, Journal of Computational and Applied Mathematics 234 (7) (2010) 2106-2117. doi:10.1016/j.cam.2009.08.069.

URL http://dx.doi.org/10.1016/j.cam.2009.08.069http://linkinghub.elsevier. com/retrieve/pii/S0377042709005469

[14] S. Lamine, M. G. Edwards, Higher-resolution convection schemes for flow in porous media on highly distorted unstructured grids, International Journal for Numerical Methods in Engineering 76 (2008) 1139-1158. doi:10.1002/nme.

[15] M. Pal, M. G. Edwards, Anisotropy favoring triangulation CVD (MPFA) finite-volume approximations, International Journal for Numerical Methods in Fluids 67 (2010) 1247-1263. doi: $10.1002 / f l d$.

[16] M. F. Wheeler, I. Yotov, A multipoint flux mixed finite element method, SIAM Journal on Numerical Analysis 44 (5) (2006) 2082-2106. doi:10.1137/050638473.

URL http://epubs.siam.org/doi/abs/10.1137/050638473

[17] M. F. Wheeler, G. Xue, I. Yotov, A Family of Multipoint Flux Mixed Finite Element Methods for Elliptic Problems on General Grids, Procedia Computer Science 4 (2011) 918-927. doi: $10.1016 / j$.procs .2011 .04 .097$.

URL http://linkinghub.elsevier.com/retrieve/pii/S1877050911001554

[18] J. Moortgat, A. Firoozabadi, Higher-order compositional modeling with Fickian diffusion in unstructured and anisotropic media, Advances in Water Resources 33 (9) (2010) 951-968. doi: $10.1016 / j$.advwatres . 2010.04.012.

URL http://linkinghub.elsevier.com/retrieve/pii/S0309170810000849

[19] J. Moortgat, Z. Li, A. Firoozabadi, Three-phase compositional modeling of CO2 injection by higher-order finite element methods with CPA equation of state for aqueous phase, Water Resources Research 48 (12) (2012) 1-21. doi:10.1029/2011WR011736.

URL http://doi.wiley.com/10.1029/2011WR011736

[20] V. R. Voller, Basic Control Volume Finite Element Methods for Fluids and Solids, IISc Press, 2009.

[21] F. S. V. Hurtado, C. R. Maliska, A. F. C. da Silva, J. Cordazzo, S. Catarina, A Quadrilateral Element-Based Finite-Volume Formulation for the Simulation of Complex Reservoirs, SPE Journal.

[22] A. S. Abushaikha, M. J. Blunt, O. R. Gosselin, C. C. Pain, M. D. Jackson, Interface control volume finite element method for modelling multi-phase fluid flow in highly heterogeneous and fractured reservoirs, Journal of Computational Physics 298 (2015) 41-61.

[23] P. Salinas, D. Pavlidis, Z. Xie, H. Osman, C. C. Pain, M. D. Jackson, A discontinuous control volume finite element method for multi-phase flow in heterogeneous porous media, Journal of Computational Physics 352 (2018) 602-614. 
[24] M. E. Hubbard, P. L. Roe, Compact high-resolution algorithms for time-dependent advection on unstructured grids, International Journal for Numerical Methods in Fluids 33 (5) (2000) 711-736. doi:10.1002/1097-0363(20000715) 33:5<711: :AID-FLD27>3.0.C0;2-0. URL http://doi.wiley.com/10.1002/1097-0363(20000715)33:5<711: :AID-FLD27>3.0. $\mathrm{CO} ; 2-0$

[25] J. E. Kozdon, B. T. Mallison, M. G. Gerritsen, Multidimensional upstream weighting for multiphase transport in porous media, Computational Geosciences 15 (2011) 399-419. doi: $10.1007 / \mathrm{s} 10596-010-9211-5$.

[26] S. Bryson, D. Levy, High-order semi-discrete central-upwind schemes for multi-dimensional Hamilton-Jacobi equations, Journal of Computational Physics 189 (1) (2003) 63-87. doi: 10.1016/S0021-9991(03)00201-8.

URL http://linkinghub.elsevier.com/retrieve/pii/S0021999103002018

[27] G. Valenti, K. Jessen, B. T. Mallison, M. G. Gerritsen, High-Order Upwind Schemes for ThreePhase Multicomponent Flows, A Preliminary Investigation, SPE Annual Technical Conference and Exhibition (2013) 1-9doi:10.2118/90594-MS.

URL http://www. onepetro.org/doi/10.2118/90594-MS

[28] W. H. Chen, L. J. Durlofsky, B. Engquist, S. Osher, Minimization of Grid Orientation Effects Through Use of Higher Order Finite Difference Methods, SPE Journal I (2) (1992) 43-52.

[29] M. Moshiri, M. T. Manzari, S. K. Hannani, A. Rasouli, Simulation of multiphase flows in porous media with gravitational effects using dominant wave method, International Journal of Numerical Methods for Heat \& Fluid Flow 23 (7) (2013) 1204-1224. doi:10.1108/ HFF-11-2010-0182.

URL http://www.emeraldinsight.com/doi/abs/10.1108/HFF-11-2010-0182

[30] H. Hoteit, A. Firoozabadi, Compositional Modeling by the Combined Discontinuous Galerkin and Mixed Methods, SPE Journal 11 (1) (2006) 19-34.

[31] A. Firoozabadi, Thermodynamics of Hydrocarbon Reservoirs, McGraw-Hill, 1999.

[32] M. L. Michelsen, The isothermal flash problem. Part I. Stability, Fluid Phase Equilibria 9 (1) (1982) 1-19.

[33] M. L. Michelsen, The isothermal flash problem. Part II. Phase-split calculation, Fluid Phase Equilibria 9 (1982) 21-40.

[34] H. Pan, A. Firoozabadi, Fast and Robust Algorithm for Compositional Modeling: Part II Two-Phase Flash Computations, SPE Journal 8 (4) (2003) 380-391.

[35] C. H. Whitson, M. R. Brulé, Phase behavior, Vol. 20, SPE, 2000.

[36] J. Lohrenz, B. Bray, C. Clark, Calculating viscosities of reservoir fluids from their compositions, SPE Journal of Petroleum Technology 16 (10) (1964) 1171-1176.

[37] M. G. Edwards, H. Zheng, S. Lamine, M. Pal, Continuous elliptic and multi-dimensional hyperbolic Darcy-flux finite-volume methods, Computers \& Fluids 46 (1) (2011) 12-22. doi: 10.1016/j.compfluid.2010.12.029.

URL http://linkinghub.elsevier.com/retrieve/pii/S0045793010003853

[38] S. Lamine, M. G. Edwards, Higher order multidimensional wave oriented upwind schemes for flow in porous media on unstructured grids, in: SPE Reservoir Simulation Symposium, 2009, pp. 1-34. doi:10.2118/119187-MS. 
[39] M. Moshiri, M. T. Manzari, A comparative study of explicit high-resolution schemes for compositional simulations, International Journal of Numerical Methods for Heat \& Fluid Flow 29 (1) (2019) 94-131. doi:10.1108/HFF-08-2017-0333.

[40] J. B. Bell, P. Colella, J. A. Trangenstein, Higher order Godunov methods for general systems of hyperbolic conservation laws, Journal of Computational Physics 82 (2) (1989) 362-397.

[41] E. F. Toro, Riemann Solvers and Numerical Methods for Fluid Dynamics: A Practical Introduction, third edit Edition, Springer, 2009.

[42] M. G. Edwards, Global and local central non-upwind finite volume schemes for hyperbolic conservation laws in porous media, International Journal for Numerical Methods in Fluids 64 (7) (2010) 793-811. doi:10.1002/fld.

[43] J. Cabello, K. Morgan, R. Loehner, A comparison of higher order schemes used in a finite volume solver for unstructured grids, in: Fluid Dynamics Conference, 1994, p. 2293.

[44] P. R. M. Lyra, K. Morgan, A Review and Comparative Study of Upwind Biased Schemes for Compressible Flow Computation. Part II: 1-D Higher-Order Schemes, Archives of Computational Methods in Engineering 7 (December 1999) (2000) 19-55.

[45] C. Hirsch, Numerical Computation of Internal and External Flows. Volume 1 Fundamentals of Computational Fluid Dynamics, Elsevier, 2007.

[46] B. Van Leer, Towards the Ultimate Conservative Difference Scheme. IV. A New Approach to Numerical Convection, Journal of Computational Physics 23 (1977) 276-299.

[47] P. K. Sweby, High Resolution Schemes Using Flux Limiters for Hyperbolic Conservation Laws, SIAM Journal on Numerical Analysis 21 (5) (1984) 995-1011.

[48] J. Qiu, C.-W. Shu, Finite Difference WENO Schemes with Lax-Wendroff-type Time Discretizations, SIAM Journal on Scientific Computing 24 (6) (2003) 2185-2198.

[49] J. Moortgat, A. Firoozabadi, Higher-order compositional modeling of three-phase flow in 3D fractured porous media based on cross-flow equilibrium, Journal of Computational Physics 250 (2013) 425-445. doi:10.1016/j.jcp.2013.05.009.

URL http://linkinghub.elsevier.com/retrieve/pii/S0021999113003409

[50] A. Danesh, PVT and Phase Behaviour of Petroleum Reservoir Fluids, Elsevier, 1998.

[51] J. A. Trangenstein, Numerical Solution of Hyperbolic Partial Differential Equations, Cambridge University Press, 2007.

[52] O. Redlich, J. N. S. Kwong, On the thermodynamics of solutions. V. An equation of state. fugacities of gaseous solutions, Chemical Reviews 44 (1) (1949) 233-244.

[53] G. Soave, Equilibrium constants from a modified Redlich-Kwong equation of state, Chemical Engineering Science 27 (6) (1972) 1197-1203.

[54] D.-Y. Y. Peng, D. B. Robinson, A New Two-Constant Equation of State, Industrial \& Engineering Chemistry Fundamentals 15 (1) (1976) 59-64. doi:10.1021/i160057a011.

URL http://pubs.acs.org/doi/abs/10.1021/i160057a011 


\section{Appendix A. Calculation of HLL Wave Speeds}

The two wave speeds of HLL scheme, i.e., $\tilde{\lambda}_{L}$ and $\tilde{\lambda}_{R}$, are defined as [51],

$$
\tilde{\lambda}_{L}=\min \left(\lambda_{L}^{\min }, \lambda_{M}^{\min }\right)
$$

and

$$
\tilde{\lambda}_{R}=\max \left(\lambda_{M}^{\max }, \lambda_{R}^{\max }\right),
$$

where $\lambda_{X}^{\max } \equiv \max _{i=1}^{n_{c}}\left(\lambda_{X i}\right)$ and $\lambda_{X}^{\min } \equiv \min _{i=1}^{n_{c}}\left(\lambda_{X i}\right)$ are the maximum and minimum of eigenvalues calculated at point $X$, respectively.

\section{Appendix B. WENO Procedure}

The procedure for obtaining weight functions in WENO scheme of (31) is as follow:

1. Find the constants $d_{r}$ for $r=0,1$, as

$$
d_{0}=\frac{2}{3}, \quad d_{1}=\frac{1}{2},
$$

2. Find the smooth indicators $\beta_{r}$ for $r=0,1$, as

$$
\beta_{0}=\left(m_{j}-m_{i}\right)^{2}, \quad \beta_{1}=\left(m_{i}-m_{I}\right)^{2},
$$

3. Form the weight $w_{r}$ for $r=0,1$, as

$$
w_{0}=\frac{\alpha_{0}}{\alpha_{0}+\alpha_{1}}, \quad w_{1}=\frac{\alpha_{1}}{\alpha_{0}+\alpha_{1}},
$$

where

$$
\alpha_{0}=\frac{d_{0}}{\left(\epsilon+\beta_{0}\right)^{2}}, \quad \alpha_{1}=\frac{d_{1}}{\left(\epsilon+\beta_{1}\right)^{2}},
$$

in which $\epsilon=10^{-6}$.

4. Calculate the $k$ reconstructed values $m_{r}$ for $r=0,1$ for each edge as,

$$
m_{0}=\frac{1}{2} m_{i}+\frac{1}{2} m_{j}, \quad m_{1}=-\frac{1}{2} m_{I}+\frac{3}{2} m_{i},
$$

5. Finally, the second order reconstruction for left state is given by

$$
m_{L}=w_{0} m_{0}+w_{1} m_{1} .
$$

The same procedure can be utilized for calculating $m_{R}$.

\section{Appendix C. Fluid Properties and Thermodynamic Equilibrium}

In order to determine phase properties, e.g., density $\rho^{\alpha}$, viscosity $\mu^{\alpha}$ and saturation $s^{\alpha}$, in a compositional model, thermodynamic (or phase) equilibrium state must be known. A multi-component multi-phase system at specified temperature and pressure is in thermodynamic equilibrium when there is no driving force between different phases of each component [50]. In this case, each phase has different composition, but all have the same temperature and pressure. Total mole fraction of components $\mathbf{z}=\mathbf{m} /\left(\mathbf{e}^{\top} \mathbf{m}\right)$ and mole fraction of components in phase $\alpha, \mathbf{x}^{\alpha}=\mathbf{m}^{\alpha} /\left(\mathbf{e}^{\top} \mathbf{m}^{\alpha}\right)$ are defined with respect to $\mathbf{m}$ and $\mathbf{m}^{\alpha}$ in section 2 . Thermodynamic equilibrium state of a multicomponent multi-phase system is the state with minimum Gibbs energy. Mathematically speaking $d G(T, p, \mathbf{z})=0$, where $G$ represents Gibbs energy [35].

In order to find out how many phases exist in the reservoir, stability analysis [32] should be performed that specifies the number of phases by evaluating different probable single- or two-phase states. Again, the actual state is the state which has the least Gibbs energy [35]. 
Whenever both liquid and vapor phases exist, flash calculation is perform to determine the composition of each phase. For a system with liquid and vapor phases, the criterion of thermodynamic equilibrium becomes [31]

$$
f_{i}^{l}\left(T, p, \mathbf{x}^{l}\right)=f_{i}^{v}\left(T, p, \mathbf{x}^{v}\right), \quad i=1, \cdots, n_{c},
$$

where $f_{i}^{\alpha}$ is partial fugacity of component $i$ in phase $\alpha$.

$f_{i}^{\alpha}$ is a complicated, non-linear function of $T, p$, and $\mathbf{x}^{\alpha}$ which is derived through using Equation Of State (EOS). In order to calculate the fugacity in the flash calculations, the EOS is employed for each phase separately. Various types of EOS may be used to this end, for example [52, 53, 54], among them Peng-Robinson [54] is used here. The latter EOS is a cubic function of phase compressibility factor $Z^{\alpha}=p /\left(\rho^{\alpha} R T\right)$ where $R$ is the universal gas constant. Consequently, the inputs of flash calculations are $T, p$, and $\mathbf{z}$; while, the outputs are $\mathbf{x}^{l}, \mathbf{x}^{v}, Z^{l}$, and $Z^{v}$. This information is used to estimate the values of $\rho^{\alpha}$ which, in turn, leads to calculation of phase saturation $s^{\alpha}$ and relative permeabilities as a functions of saturation. In order to calculate phase viscosities $\mu^{\alpha}$, algorithm of [36] is used, which is briefly described in Appendix C.2. Details of PVT calculations can be found in [35].

\section{Appendix C.1. Flash Procedure}

1. Given the overall composition $z_{i}, K_{i}$ is defined as the ratio of components mole fraction in vapor to liquid phases; then, the so-called Rachford-Rice equation [32]

$$
F\left(\beta_{v}\right)=\sum_{i=1}^{n_{c}} \frac{z_{i}\left(K_{i}-1\right)}{1+\left(K_{i}-1\right) \beta_{v}}=0
$$

should be solved iteratively, with an initial guess from stability analysis, to determine the mole fraction of vapor phase $\beta_{v}=\mathbf{e}^{\top} \mathbf{m}^{v} / \mathbf{e}^{\top} \mathbf{m}$.

2. The mole fraction of components in the liquid phase is solved from the overall material balance equation as

$$
x_{i}^{l}=\frac{z_{i}}{1+\left(K_{i}-1\right) \beta_{v}},
$$

and $x_{i}^{v}=K_{i} x_{i}^{l}$.

3. Given the $T, p, \mathbf{x}^{l}$, and $\mathbf{x}^{v}$, EOS is solved for both liquid and vapor phases, and the fugacities of each component in each phase is calculated. Finally, the $K_{i}$ in part (1) are updated successively as

$$
K_{i}=K_{i}^{o l d} f_{i}^{l} / f_{i}^{v},
$$

until $\sum_{i=1}^{n_{c}}\left|f_{i}^{l}-f_{i}^{v}\right| \leq \varepsilon$ within a preset tolerance, here $\varepsilon=10^{-12}$.

It should be noted that, the properties needed for EOS calculations are given in Tables 7 .

\section{Appendix C.2. Calculation of Viscosity}

Lohrenz et al. [36] proposed the following formula for approximation of viscosity in hydrocarbon mixtures as a function of temperature, pressure, and composition:

$$
\begin{aligned}
\mu=\mu^{*}+ & 0.18383\left(\frac{M_{w}^{3} p_{p c}^{4}}{T_{p c}}\right)^{0.16667} \\
& \times\left[\left(0.1023+0.023364 \varrho_{r}+0.058533 \varrho_{r}^{2}-0.040758 \varrho_{r}^{3}+0.0093724 \varrho_{r}^{4}\right)^{4}-1.10^{-4}\right],
\end{aligned}
$$

where $M_{w}$ is molecular weight, $\varrho_{r} \equiv \frac{\varrho}{\varrho_{p c}}$ is reduced mass density while $p_{p c}, T_{p c}$, and $\varrho_{p c}$ are the so-called pseudo critical pressure, pseudo critical temperature, and pseudo critical mass density of mixture, respectively. It should be noted that the pressure unit in equation (C.5) is atmosphere 
(atm) and viscosity comes in centi-poise $(\mathrm{cP})$ unit. Pseudo critical properties are calculated using Kay's mixing rule [50] as

$$
\begin{aligned}
T_{p c} & =\sum_{i=1}^{n_{c}} x_{i} T_{c r, i}, \\
p_{p c} & =\sum_{i=1}^{n_{c}} x_{i} p_{c r, i}, \\
\frac{1}{\varrho_{p c}} & =\sum_{i=1}^{n_{c}} x_{i} \frac{1}{\varrho_{c r, i}} .
\end{aligned}
$$

Here, $x_{i}$ is the mole fraction of $i$ th component in the mixture while $p_{c r, i}, T_{c r, i}$, and $\varrho_{c r, i}$ are critical pressure, critical temperature, and critical mass density of $i$ th component, respectively. Moreover, in equation (C.5), $\mu^{*}$ is viscosity of mixture at atmospheric pressure calculated as

$$
\mu^{*}=0.18383 \frac{\sum_{i=1}^{n_{c}} x_{i} \mu_{i}^{*} \sqrt{M_{w, i}}}{\sum_{i=1}^{n_{c}} x_{i} \sqrt{M_{w, i}}}
$$

where $M_{w, i}$ is the molecular weight of $i$ th component while $\mu_{i}^{*}$ is defined as

$$
\mu_{i}^{*}= \begin{cases}3.4 \times 10^{-4} T_{r, i}^{0.94}\left(\frac{M_{w, i}^{3} p_{c r, i}^{4}}{T_{c r, i}}\right)^{0.16667} & T_{r i}<1.5 \\ 1.778 \times 10^{-4}\left(4.58 T_{r, i}-1.67\right)^{0.625}\left(\frac{M_{w, i}^{3} p_{c r, i}^{4}}{T_{c r, i}}\right)^{0.16667} & T_{r i} \geq 1.5\end{cases}
$$

in which $T_{r, i} \equiv \frac{T}{T_{c r, i}}$ is the reduced temperature. 